Status and upgrade of the

\title{
LHCb Vertex Locator
}

\author{
Marco Gersabeck \\ The University of Manchester \\ on behalf of the LHCbVELO group \\ ICATPP - Como - 25 September 2013
}




\section{$\mathrm{LHCb}$}

- General purpose experiment in the forward region

$\Rightarrow$ Heavy flavour physics: beauty and charm

$\Rightarrow$ Forward electroweak physics

$\Rightarrow$ Central exclusive production

- Long-lived exotica

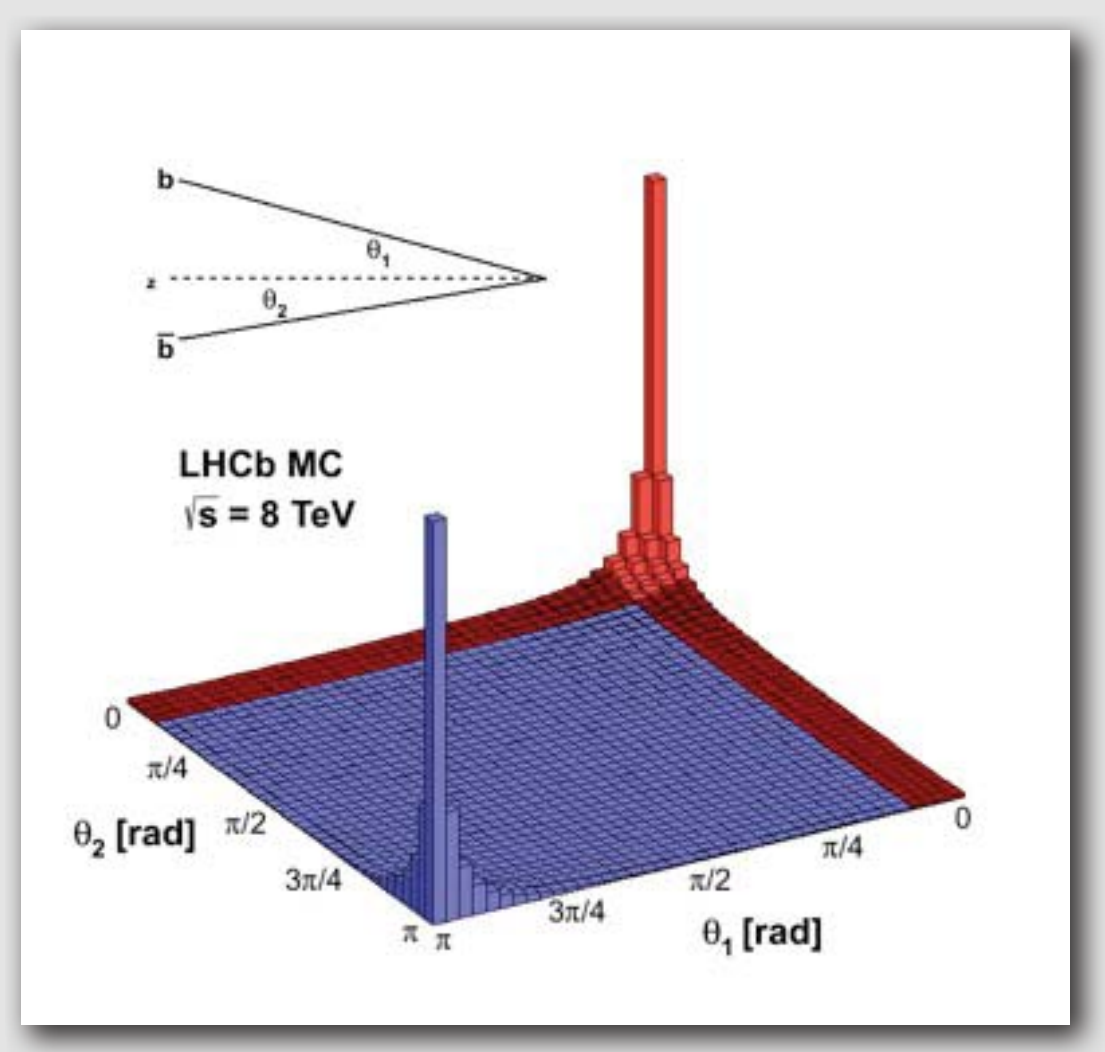


$\rightarrow$ M. Martinelli - LHCb Tracking and Alignment - Tracker and applications I (III)

$\rightarrow$ E. Gersabeck - LHCb Outer Tracker - Tracker and applications I (III)

\section{$\mathrm{LHCb}$ detector}

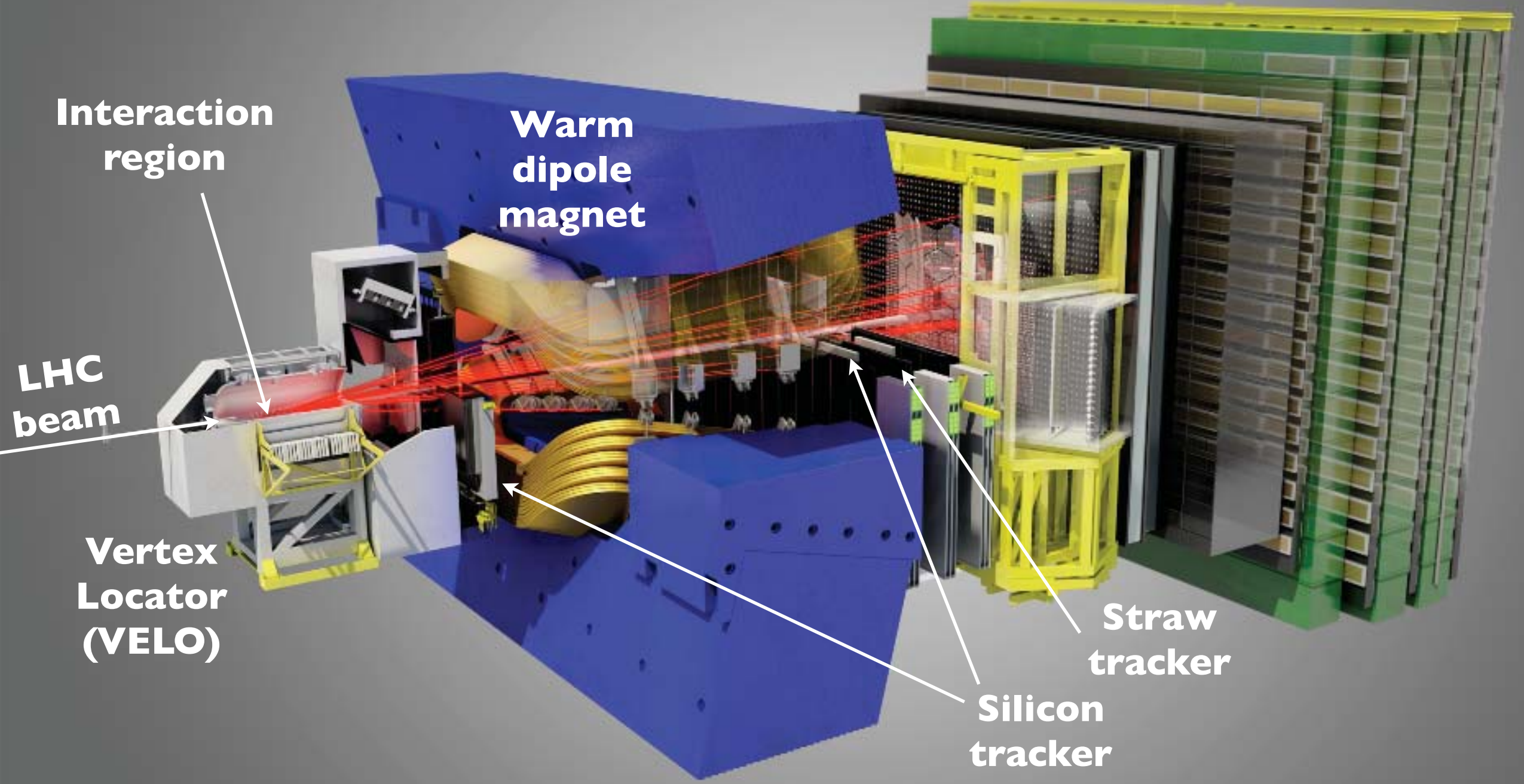


$\rightarrow$ M. Martinelli - LHCb Tracking and Alignment - Tracker and applications I (III)

$\rightarrow$ E. Gersabeck - LHCb Outer Tracker - Tracker and applications I (III)

\section{$\mathrm{LHCb}$ detector}

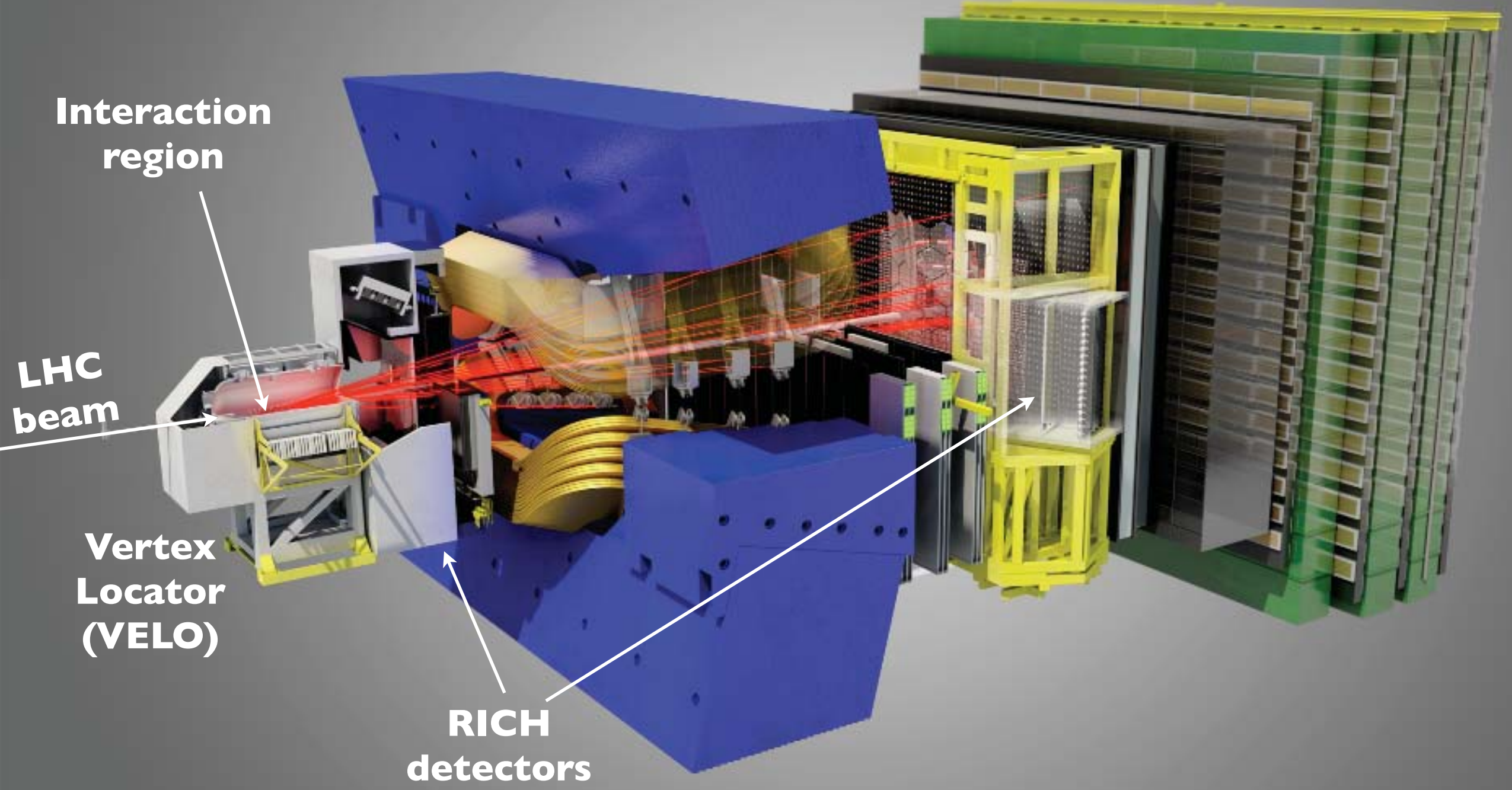


$\rightarrow$ M. Martinelli - LHCb Tracking and Alignment - Tracker and applications I (III)

$\rightarrow$ E. Gersabeck - LHCb Outer Tracker - Tracker and applications I (III)

\section{$\mathrm{LHCb}$ detector}

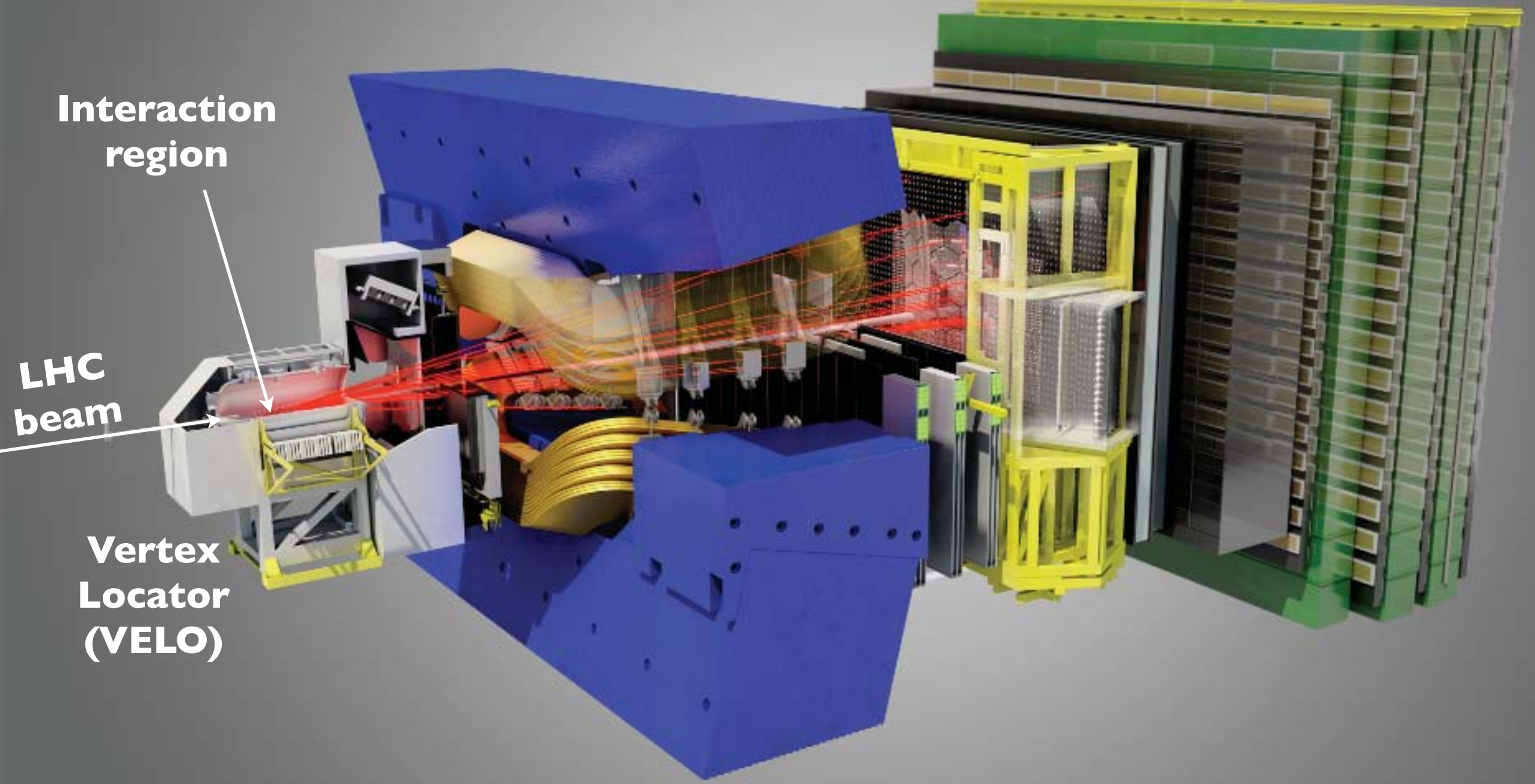


MANCHESTER 
- $300 \mu \mathrm{m}$ n-on-n sensors

*2 n-on-p sensors

- 2048 strips per sensor

- $\mathrm{R}$ and $\Phi$ geometries

- Pitch varying from $\sim 40 \mu \mathrm{m}$ to $\sim 100 \mu \mathrm{m}$

- Stereo angle for $\Phi$ strips

- Inner edge: $r=7 \mathrm{~mm}$

$\Rightarrow$ Active region edge: $r=8.2 \mathrm{~mm}$

- Second metal layer routing lines (see later)

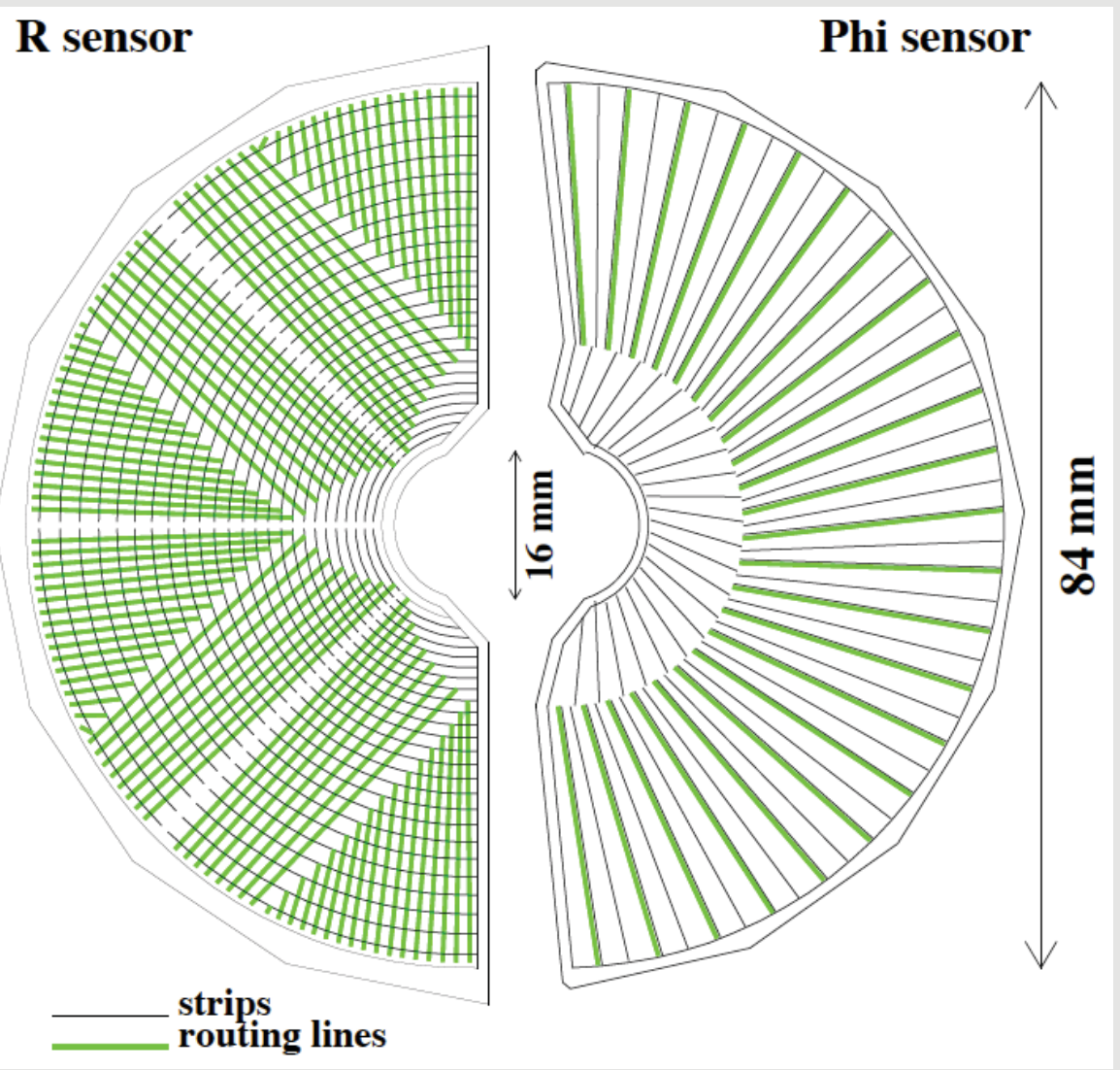




\section{MANCHESTER}

VELO performance
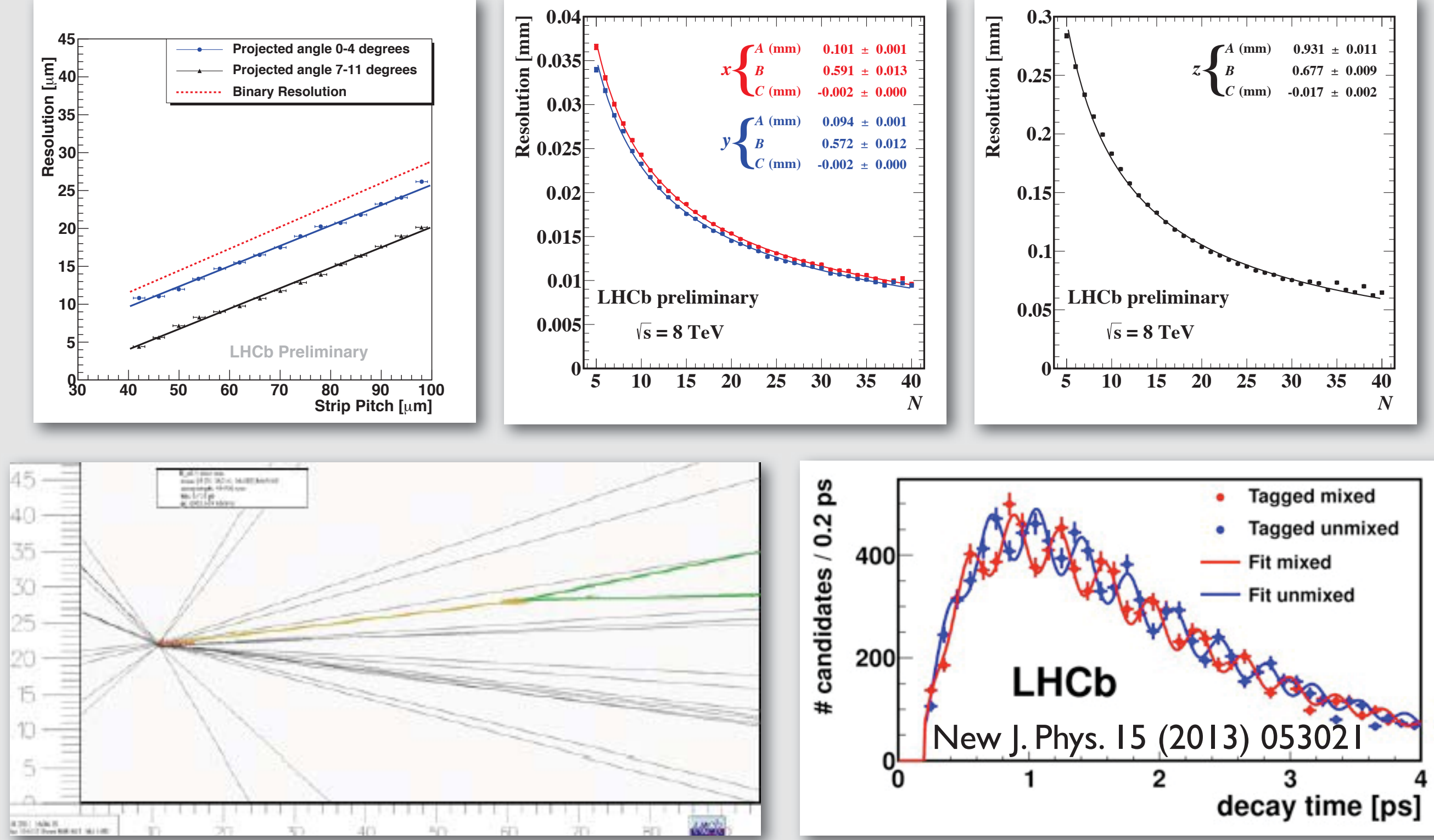


\section{MANCHESTER}

\section{4}

\section{VELO radiation damage}
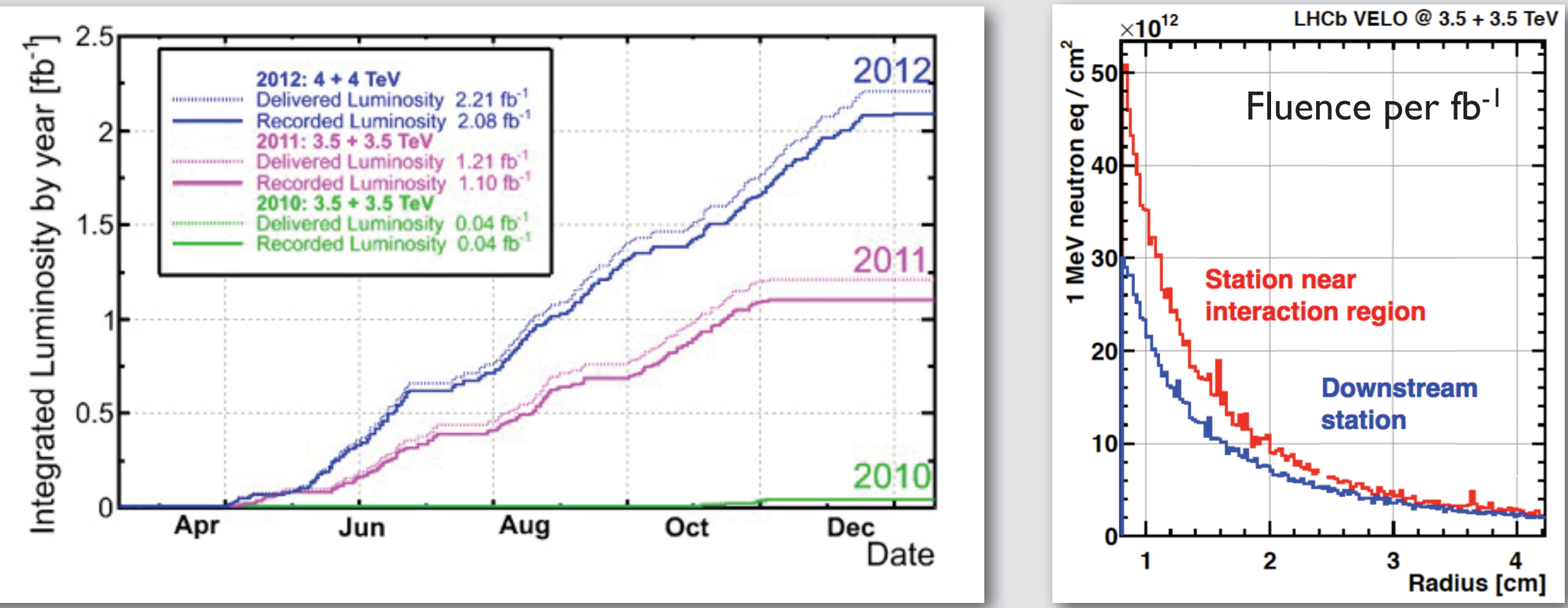

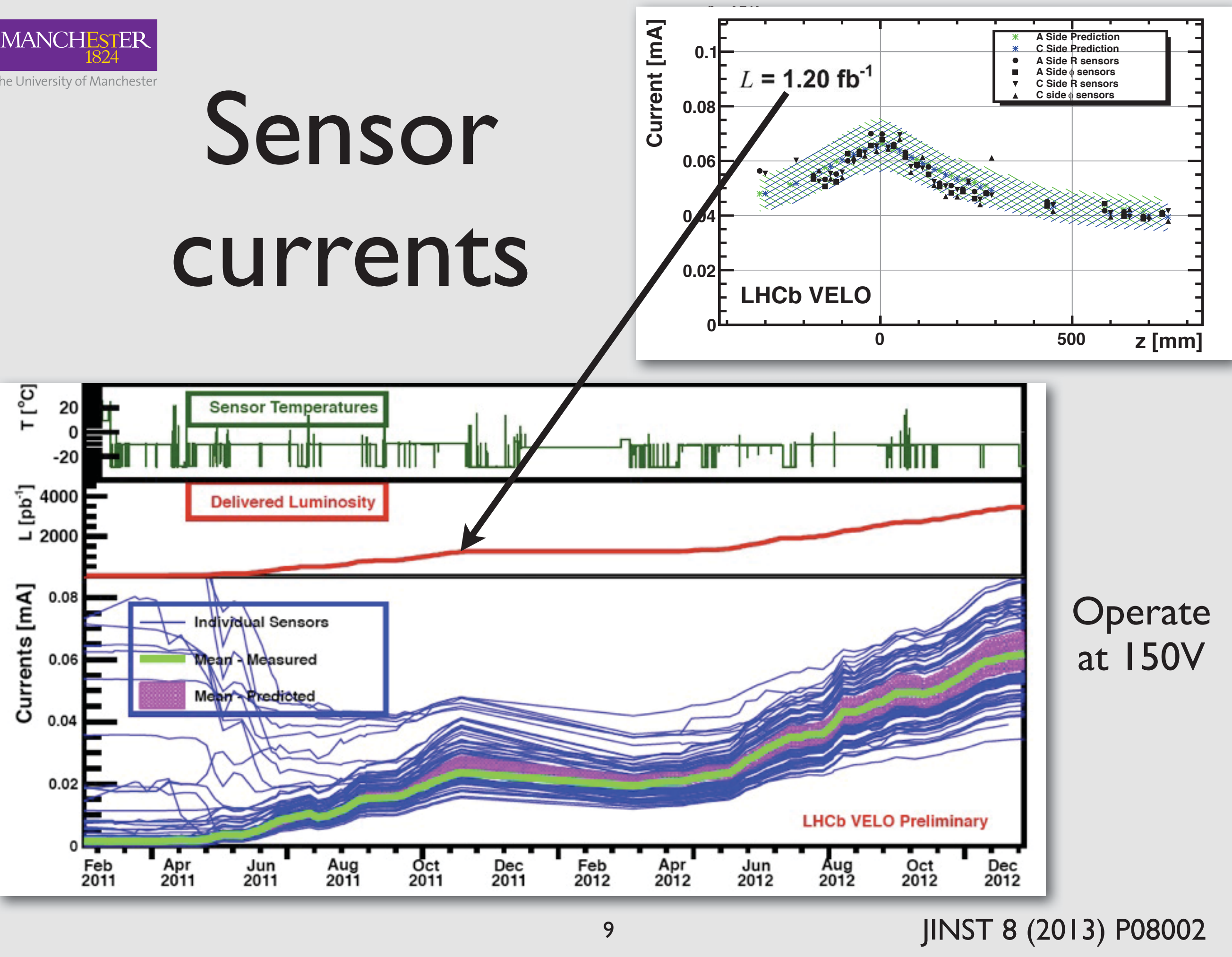


\section{Efficiency scans}

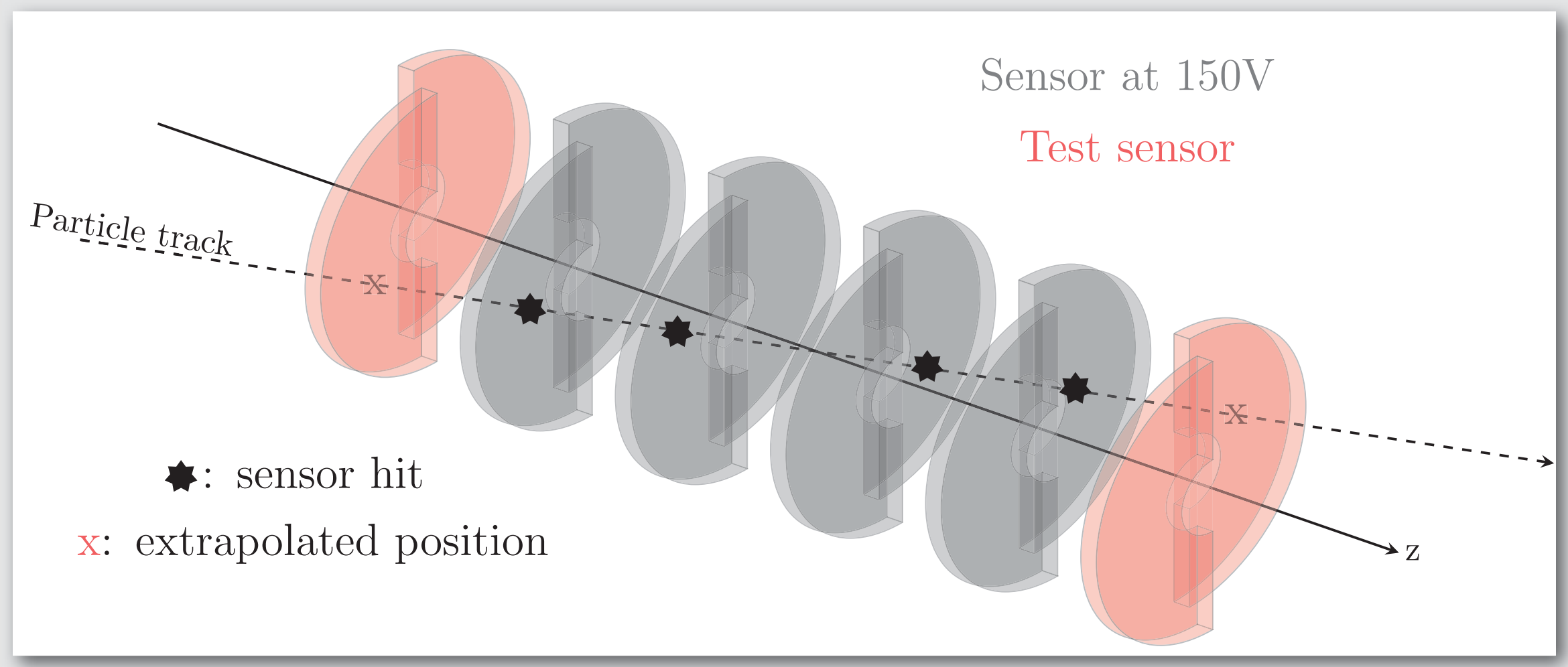

- Scan bias voltage on every $5^{\text {th }}$ module

- Allows determination of cluster efficiencies

- Competes with physics data taking: few scans per year 


\section{MANCHESTER}

Effective depletion voltage
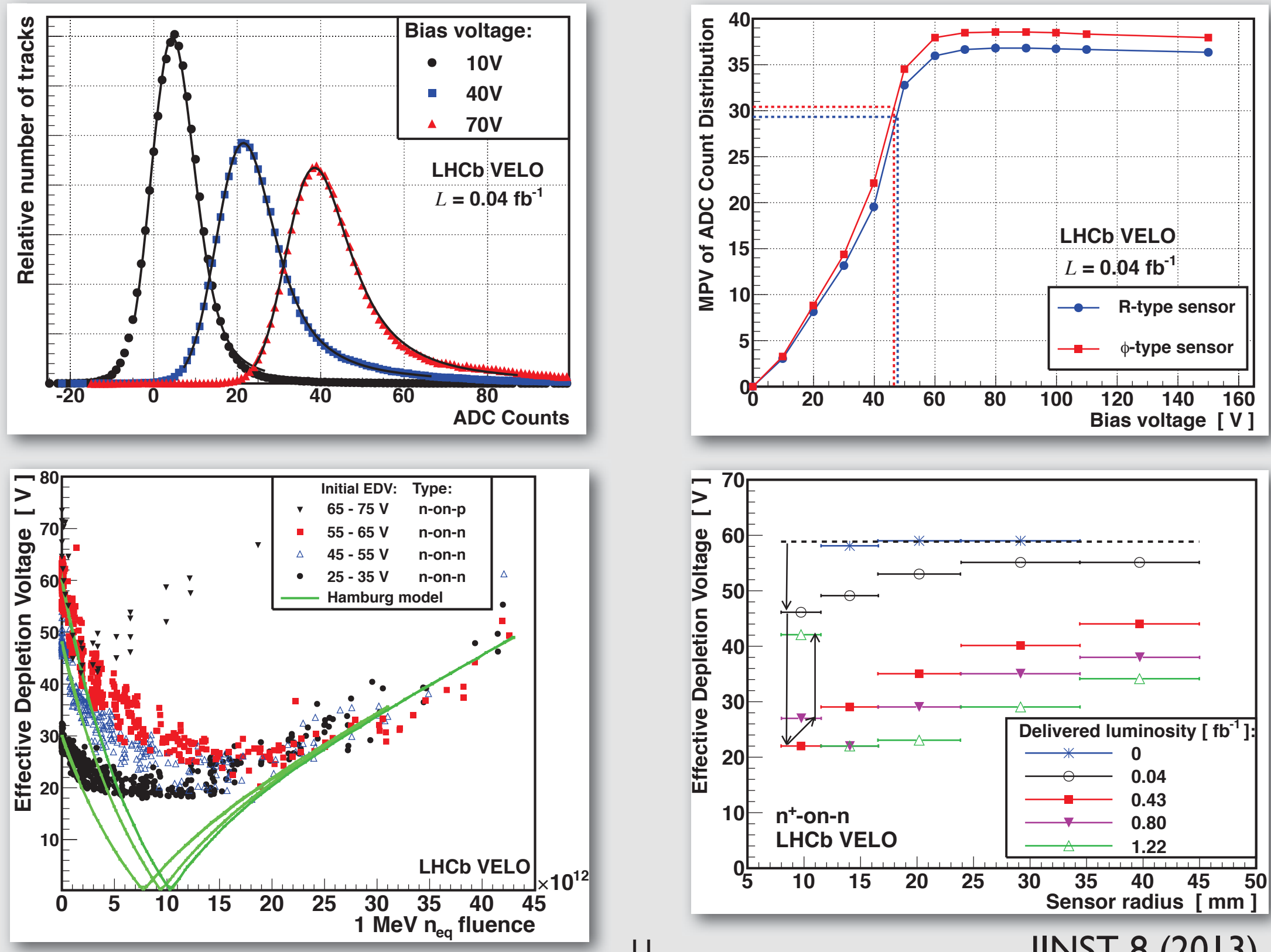


\section{MANCHESTER}

\section{$2^{\text {nd }}$ metal layer}
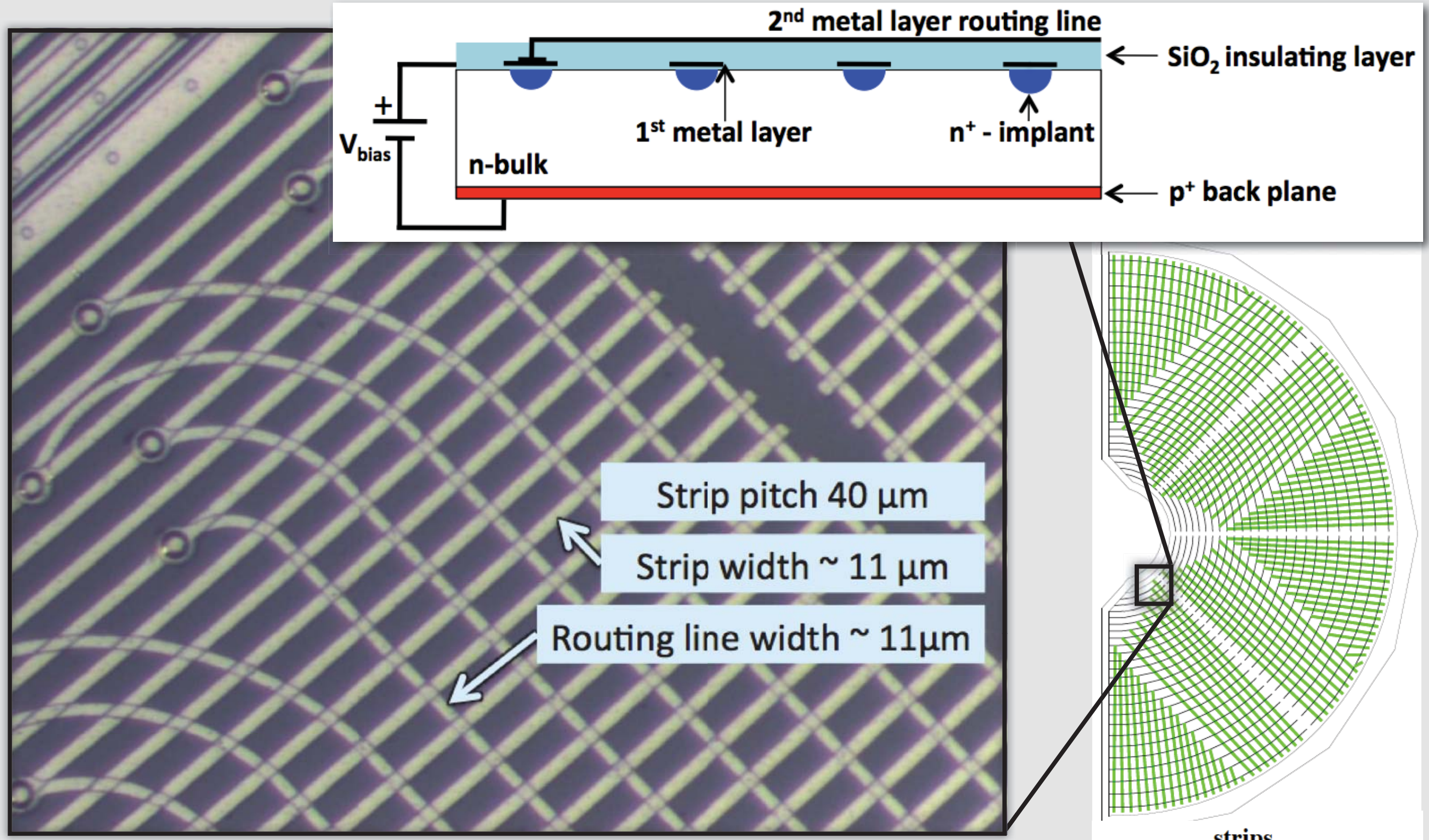

JINST 8 (20 I3) P08002

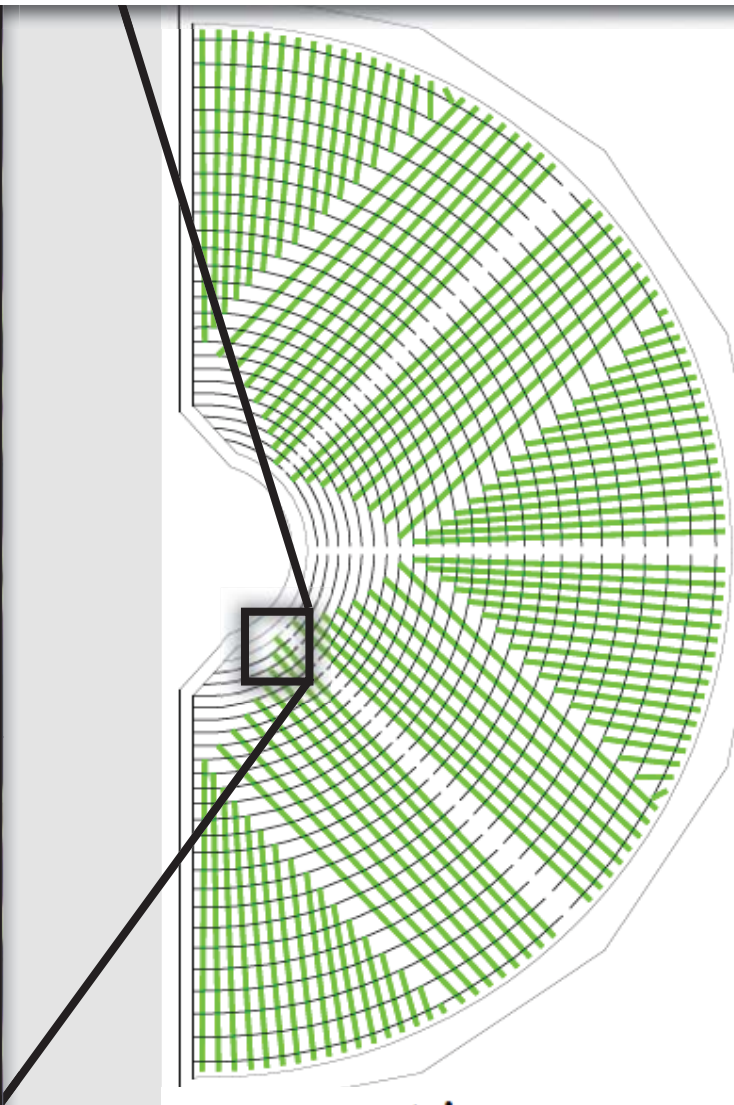

strips routing lines 


\section{MANCHESTER}

\section{$2^{\text {nd }}$ metal layer}
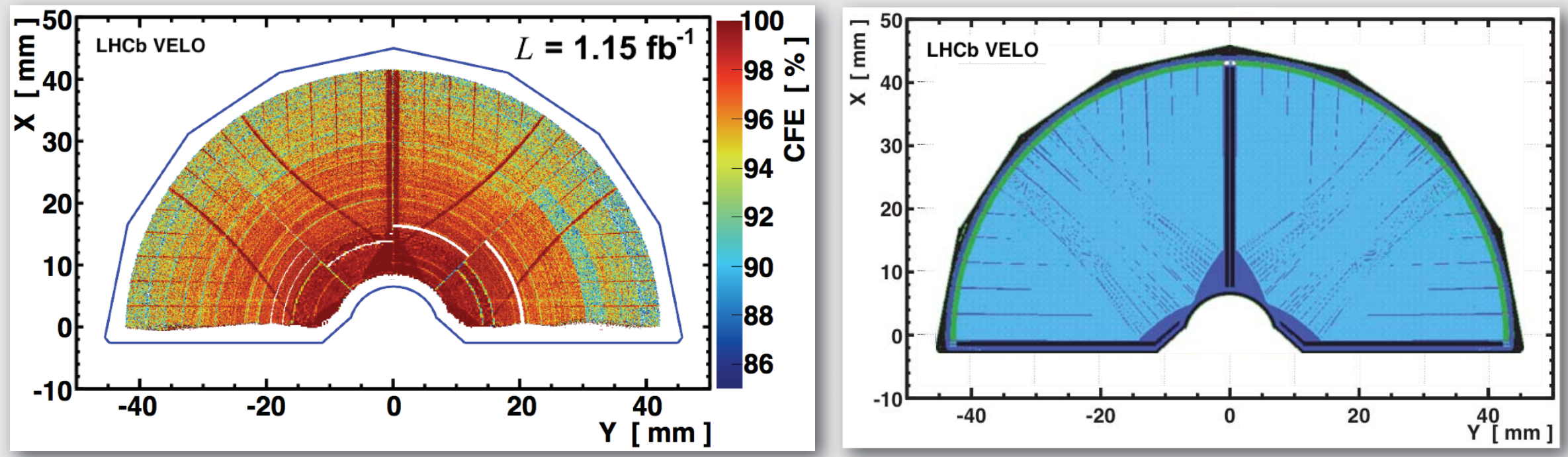

- Lowered cluster finding efficiency at large radii

- $-100 \%$ efficiency in regions without routing lines

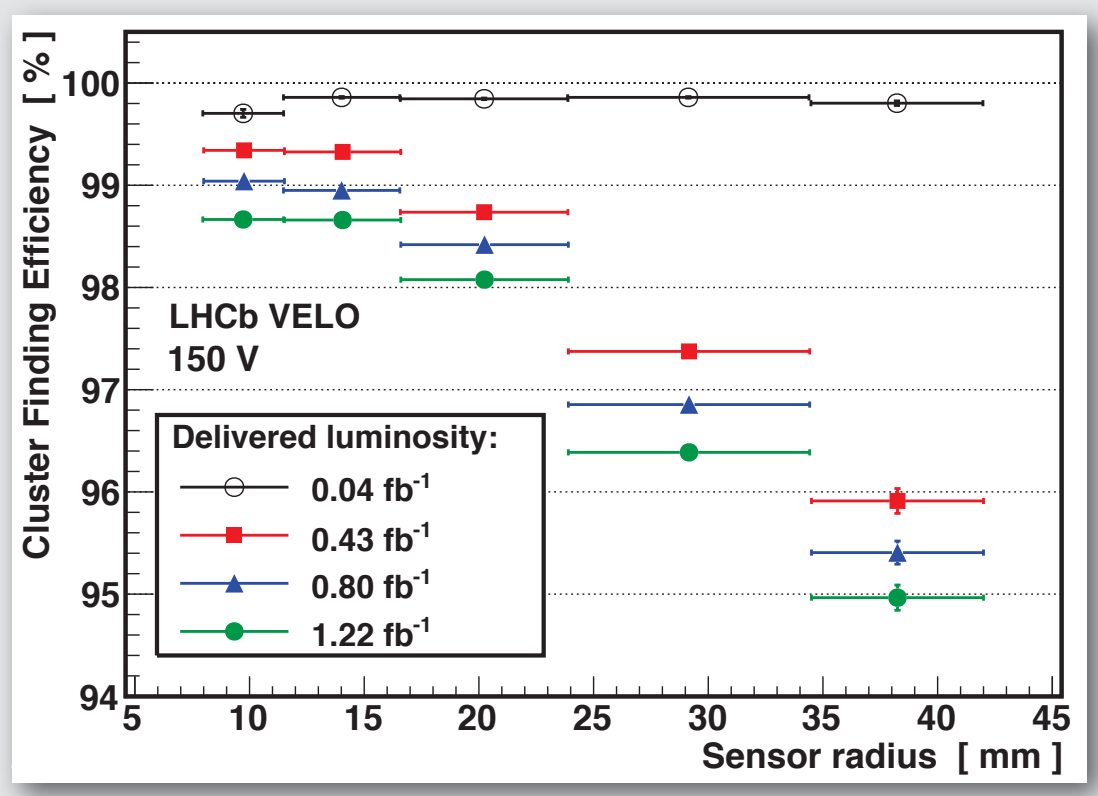




\section{MANCHESTER}

The University of Manchester
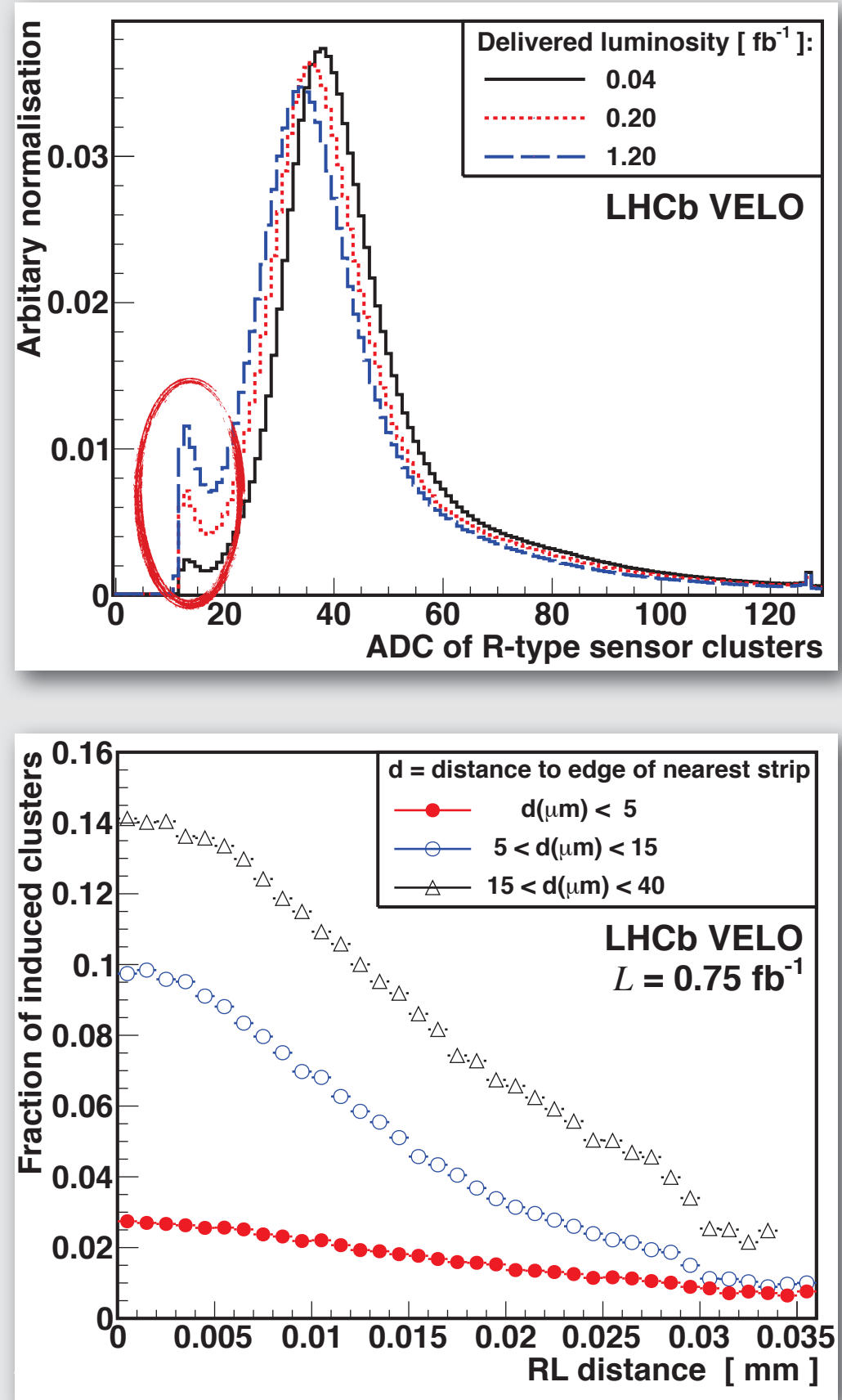

\section{$2^{\text {nd }}$ metal layer}

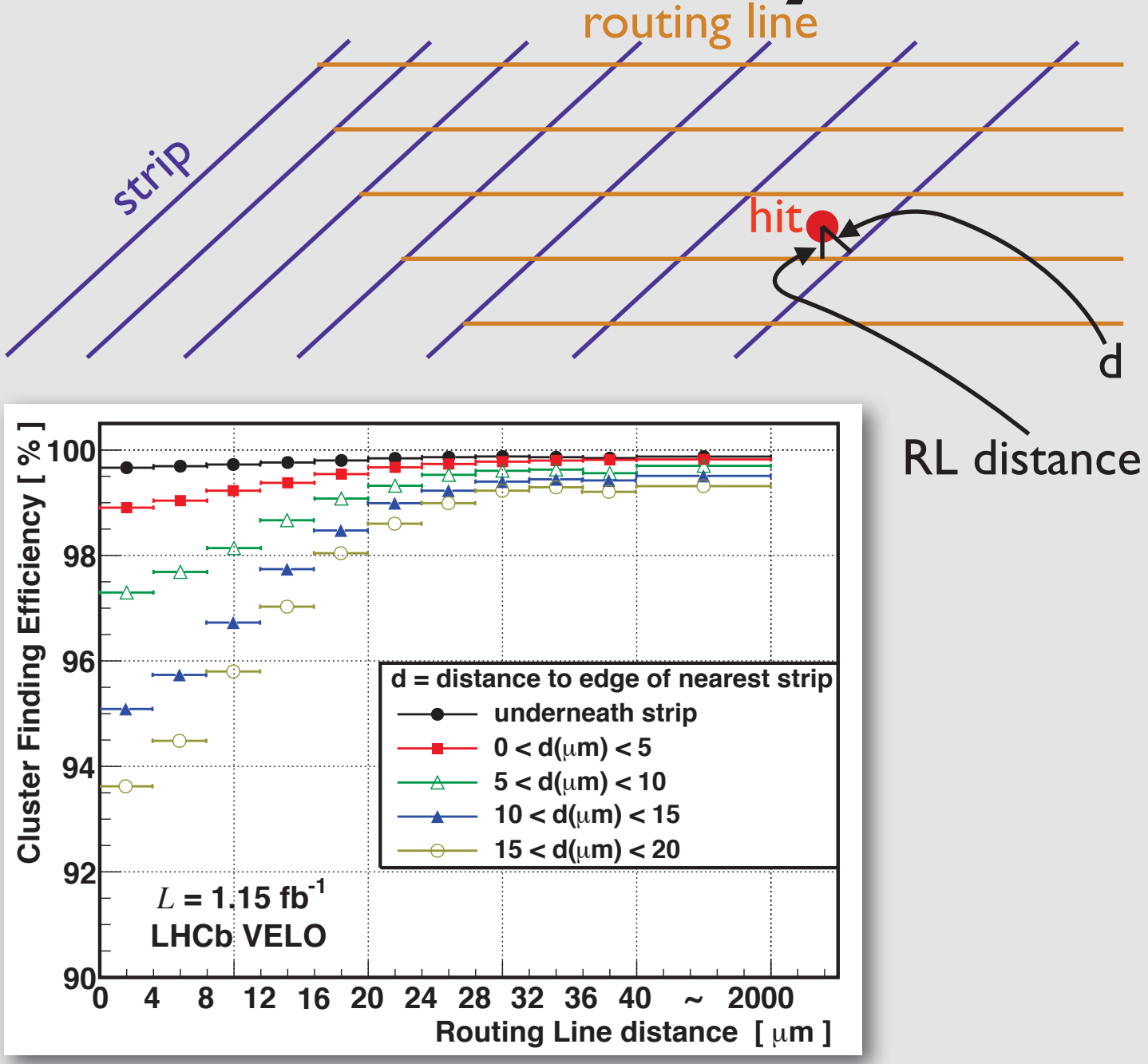

- Cluster efficiency affected

- Tracking efficiency OK 
Towards LHC run 2

- Replacement detector completed

$\Rightarrow$ Insurance for beam incidents

$\Rightarrow$ Spare if current detector has too much radiation damage

- Further streamlined operation

$\Rightarrow 2$ VELO shifters 24/7

+ I on-call expert in 2010

$\Rightarrow 2$ on-call experts in 2012

$\Rightarrow$ I on-call expert in 2015
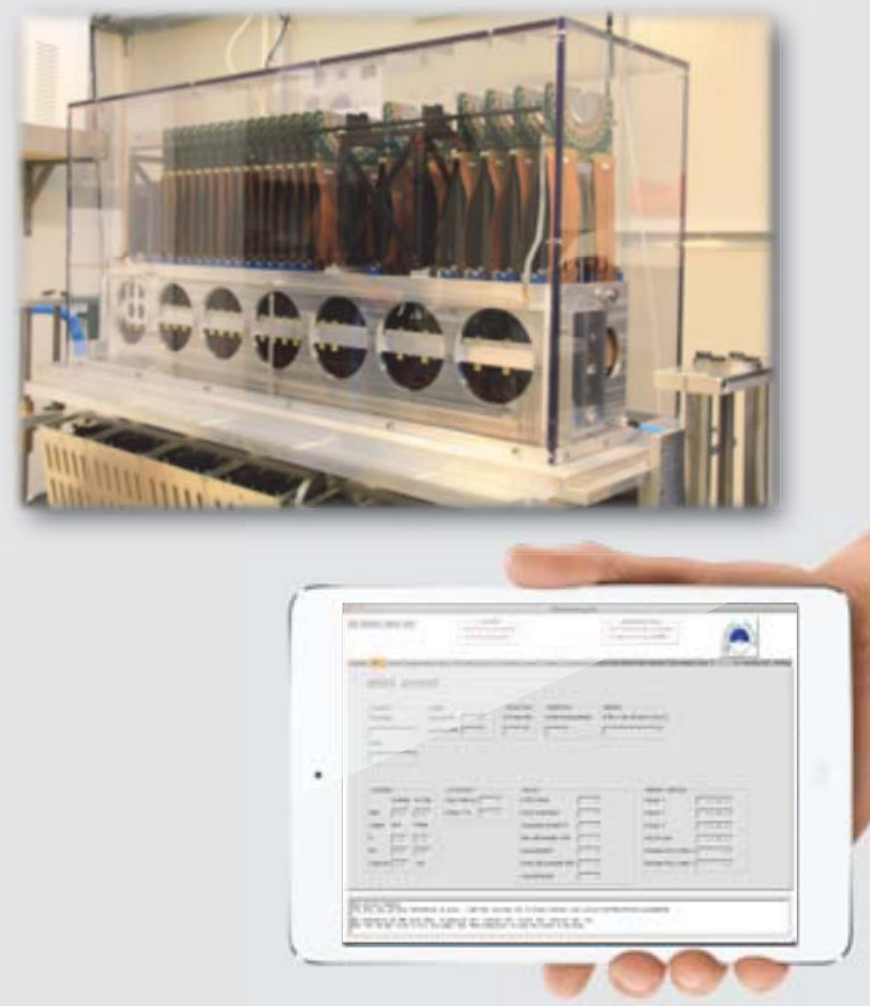
MANCHESTER 


\section{LHCb upgrade}

- Huge physics potential beyond current $\mathrm{LHCb}$ reach

- Need higher luminosity

$\Rightarrow$ now $4 \times 10^{32} \mathrm{~cm}^{-2} \mathrm{~s}^{-1}$

$\Rightarrow$ upgrade $20 \times 10^{32} \mathrm{~cm}^{-2} \mathrm{~s}^{-1}$

- Trigger limitation

$\Rightarrow$ particularly for hadronic final states

- Change from I MHz to full $40 \mathrm{MHz}$ readout

- Pile up increases from $\sim 2$ to $<6$

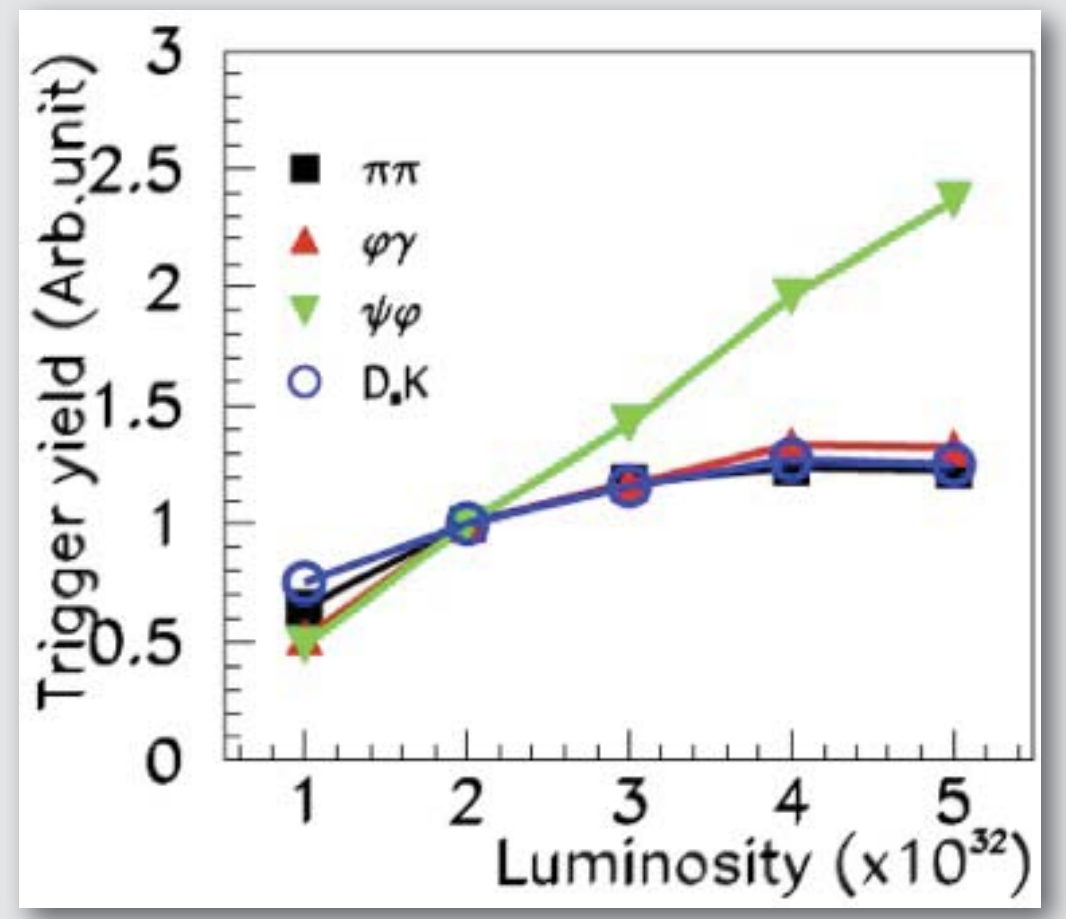

- Installation 2018: LHC long shutdown 2 
sensor unit with 3 ASICs

4 units per module ( 2 per side) inner edge at $5 \mathrm{~mm}$ radius

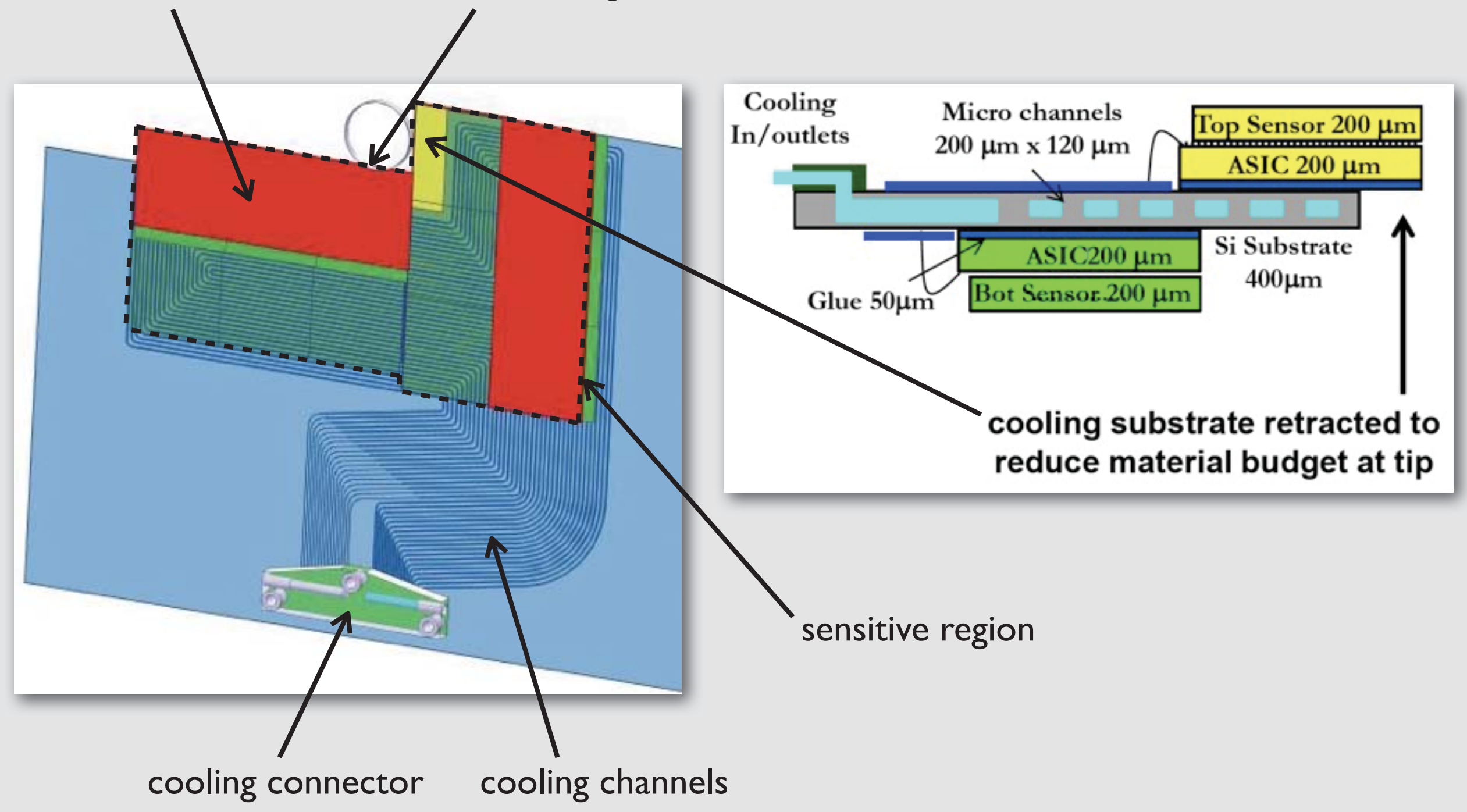




\section{Material budget}

- Low material budget $\rightarrow$ better performance

- Thinned sensors

- Thinned ASICs

- Retracted substrate

- Low-mass bases

- Thin RF shield

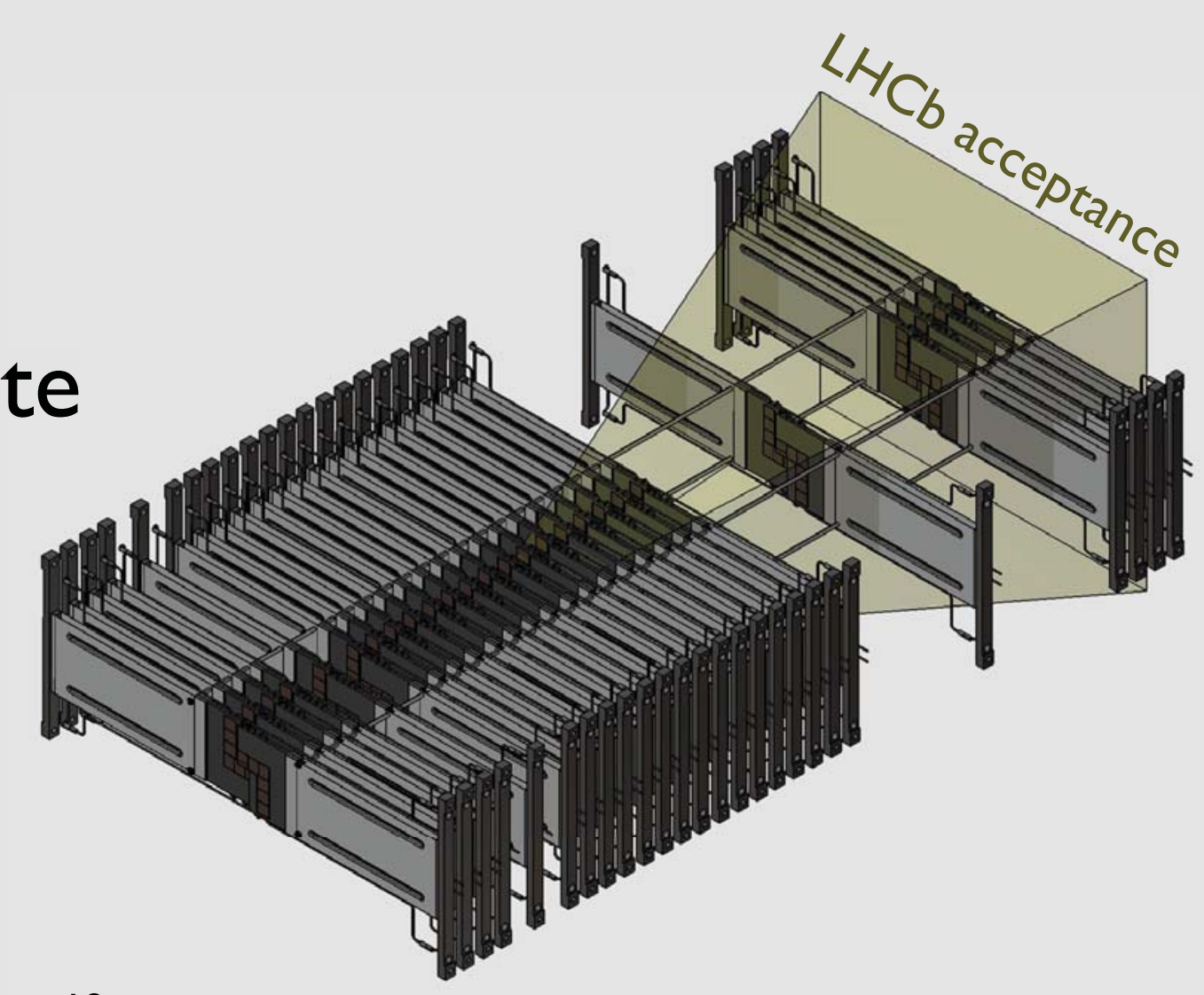


- Dual use-case:

$\Rightarrow$ RF-field guidance \& vacuum barrier

- New design:

$\Rightarrow$ L-shaped modules \& closer to beam

- New procedures to minimise thickness

$\Rightarrow$ Current VELO: pressed Aluminium, $300 \mu \mathrm{m}$ thickness

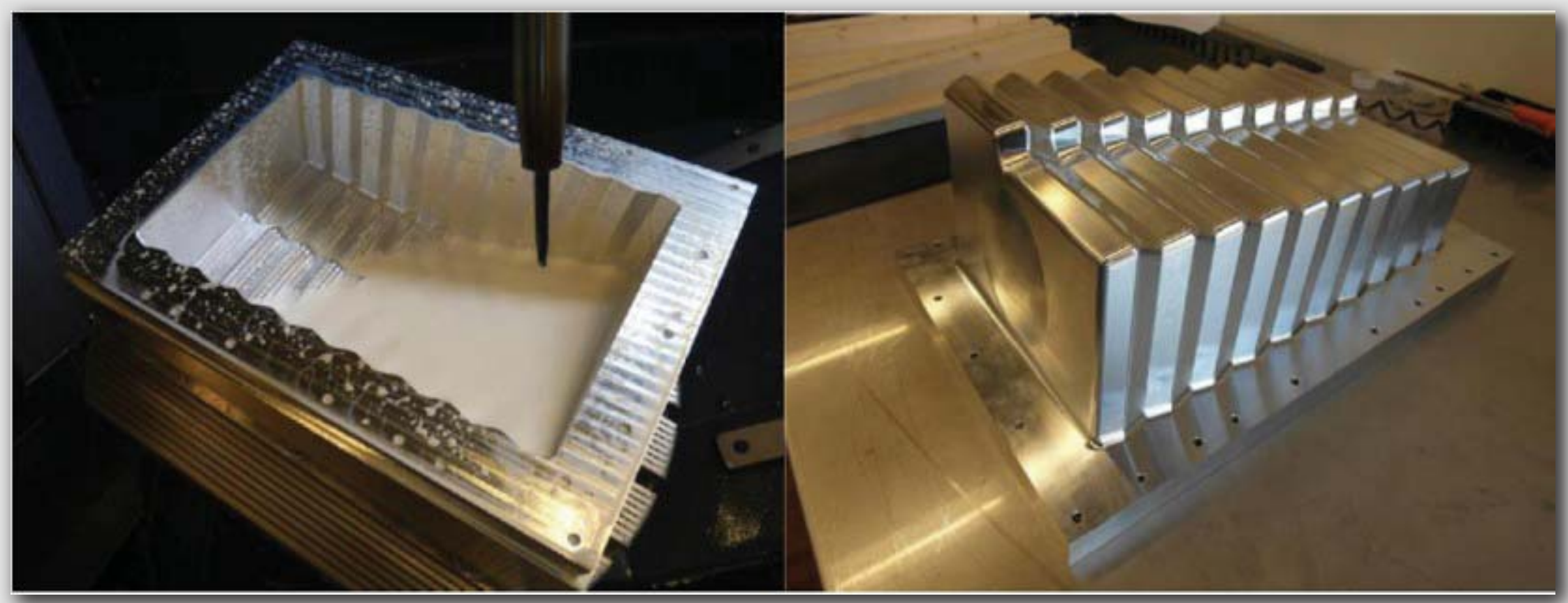


First prototypes
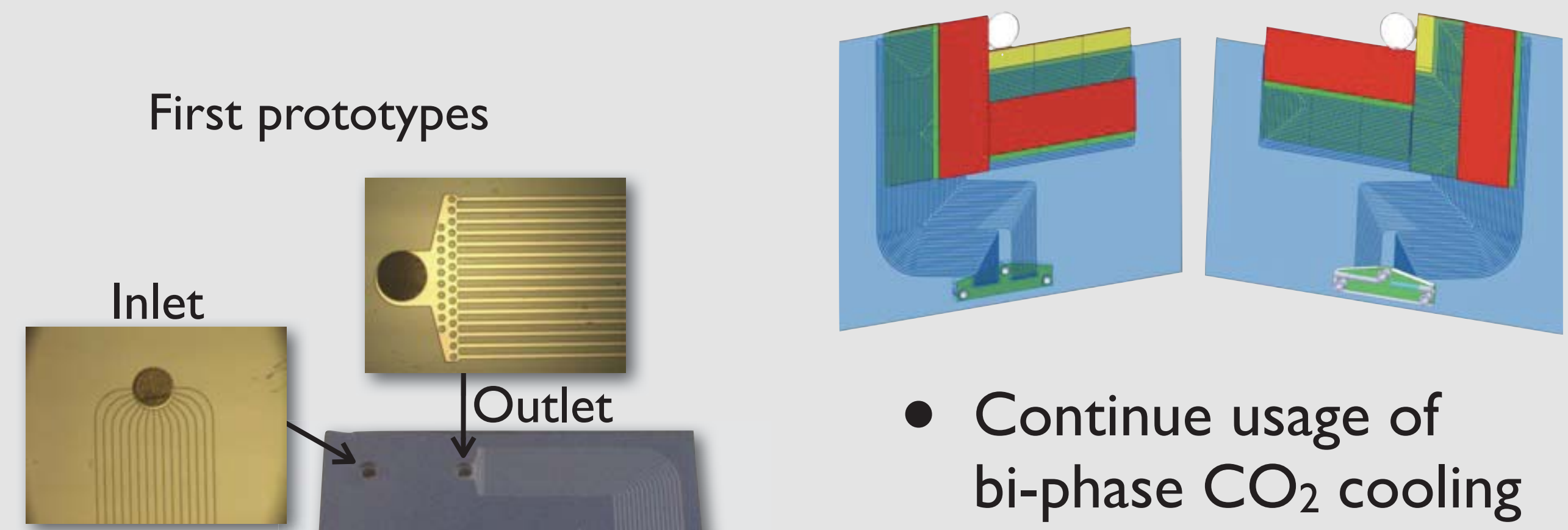

- Continue usage of bi-phase $\mathrm{CO}_{2}$ cooling

- Use microchannels to cool at heat source

\section{Transition}

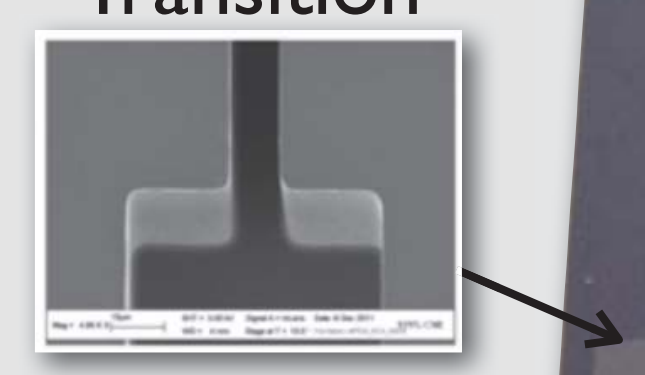

- Main challenge: mechanical stability

- Detectors in vacuum 


\section{Cooling}

- Operation

$\Rightarrow$ I5 bar at $-30^{\circ} \mathrm{C}$

$\Rightarrow 60$ bar at room temp.

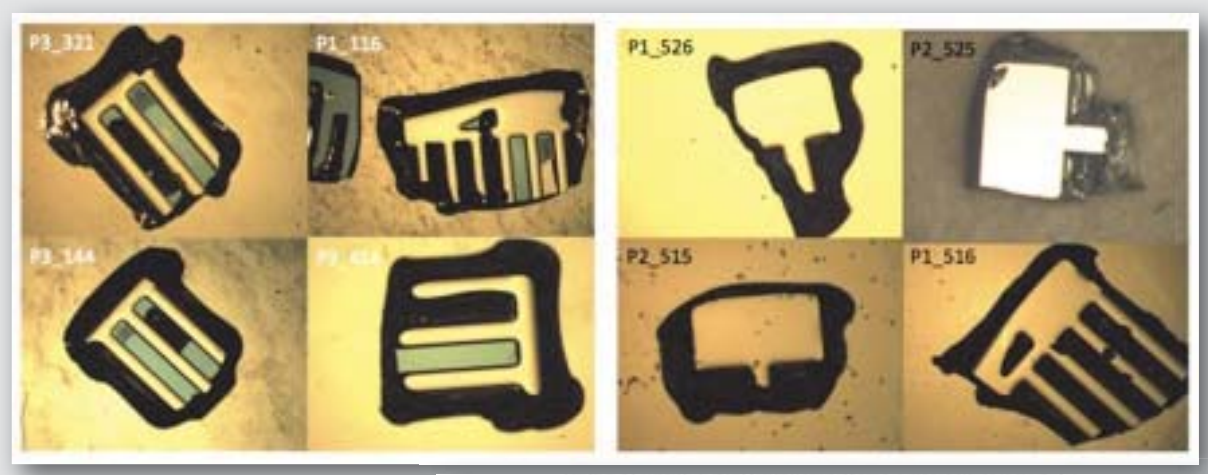

Max and Min Temperature Pressure on the C3_312

- Required safety margin I50 bar

- Latest samples use hydrophobic bonding

- Withstand 700 bar (pump limit)

- Cycling tests $\pm 40^{\circ} \mathrm{C} \& 200$ bar
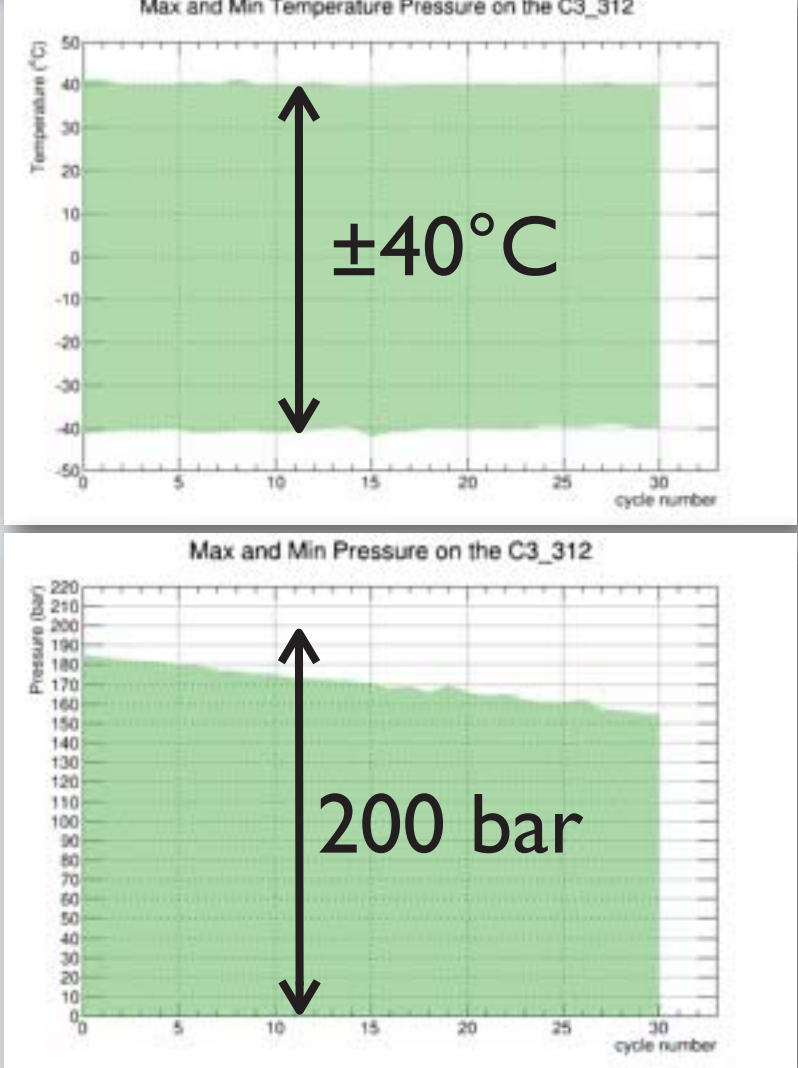


\section{Readout rates}

Avg. \#particles/chip/event

- Average (peak) event rate: 26.8 (40) $\mathrm{MHz}$

- Hottest chip: 230 (320) Mtracks/s/chip

- Hit rate: 600 (890) MHits/s/chip

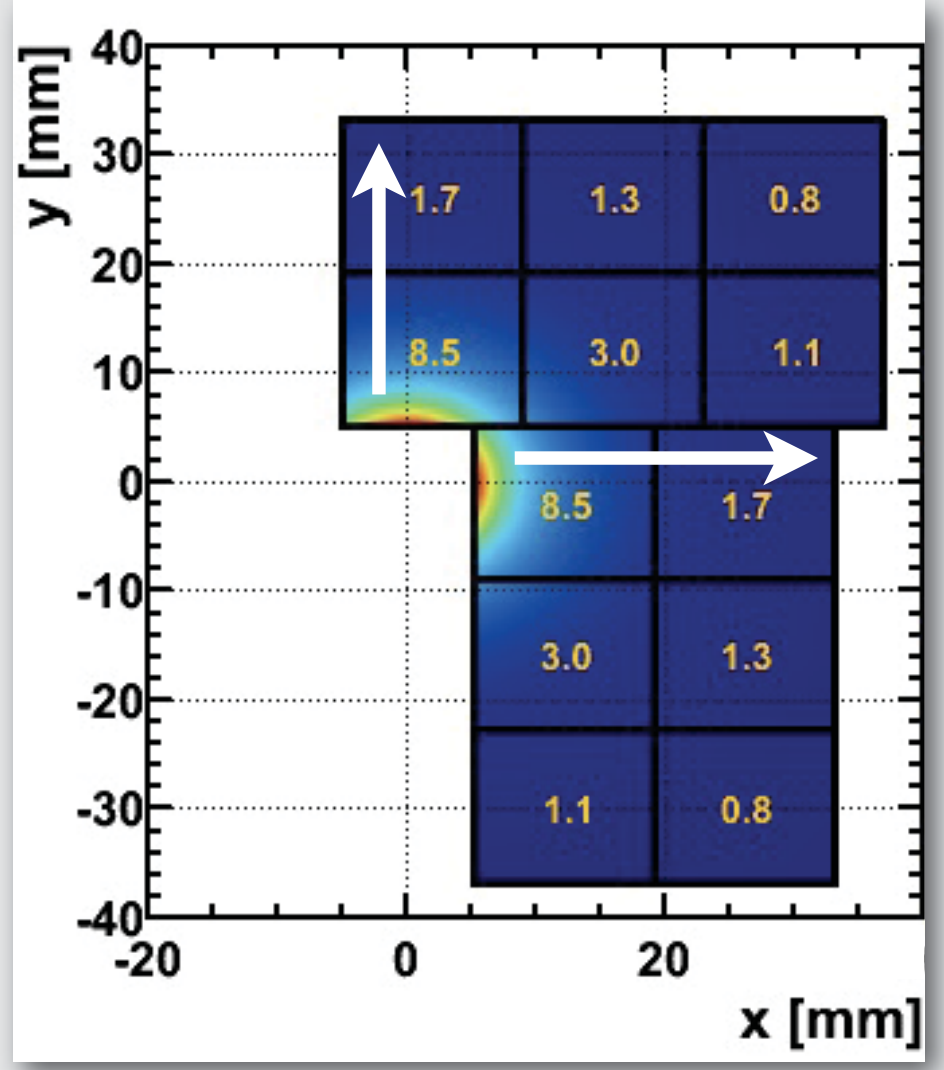


- Front end chip:

VeloPix based on Timepix3

- $<3$ W/chip

- Grouping to increase rate capability

$\Rightarrow 2 \times 4$ super pixels

- 4 links of $5 \mathrm{Gbit} / \mathrm{s}$

- First submission summer 2014

- Production end 2015

Timepix3

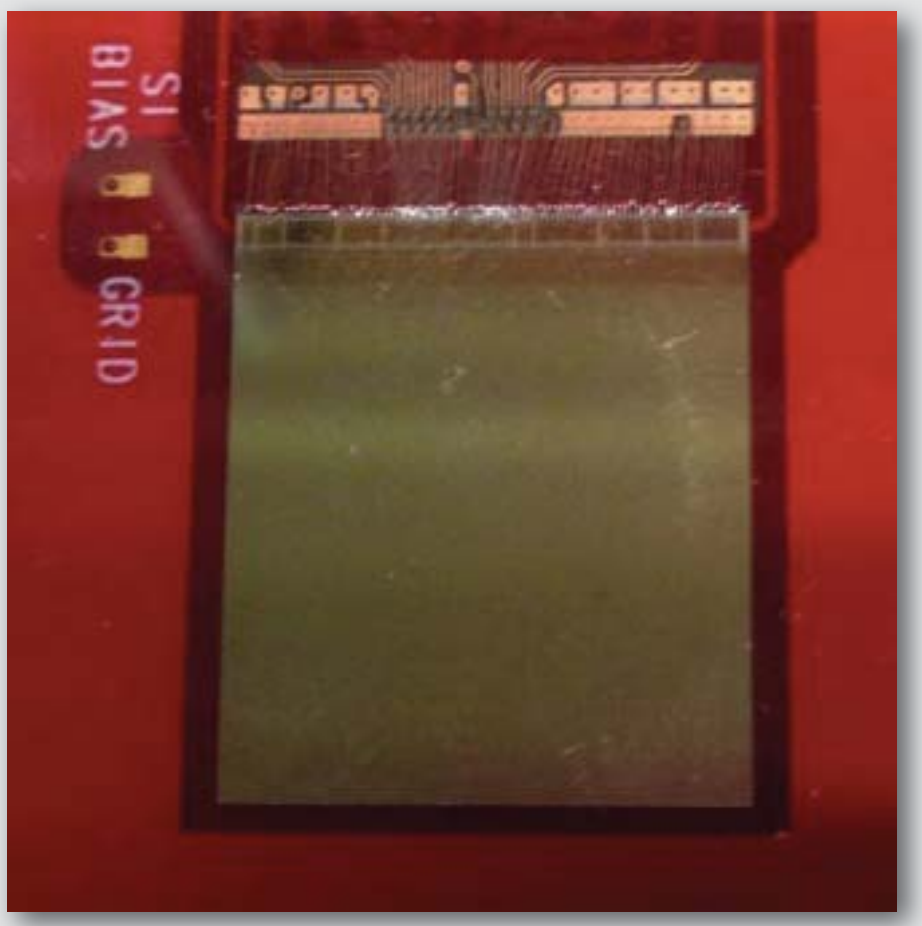




\section{Readout chain}

- Optical transmission from detector to readout boards

- FPGA-based DAQ boards

- Feed into trigger farm

$\Rightarrow$ Full event information at $40 \mathrm{MHz}$

$\Rightarrow$ Software trigger

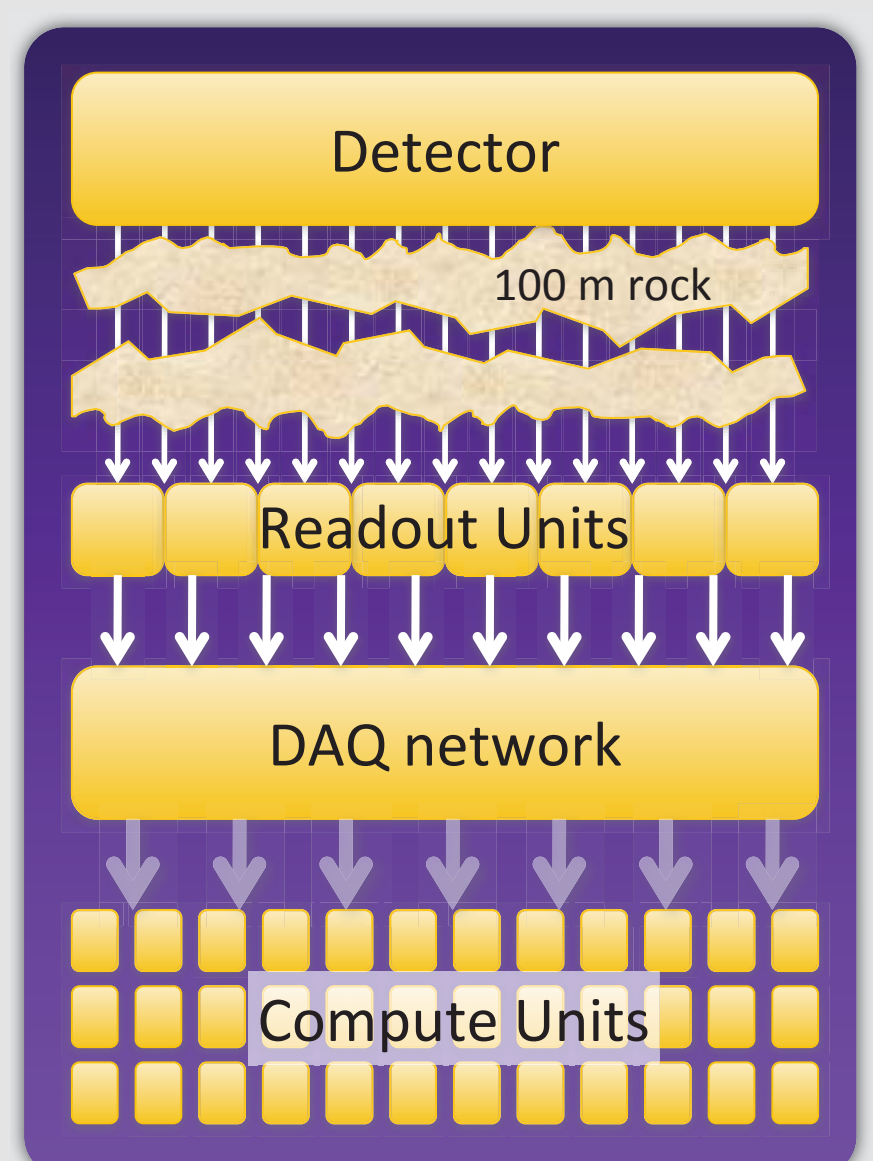




\section{Performance}

- Improved impact parameter resolution

$\Rightarrow$ Reduced inner radius

$\Rightarrow$ Optimised material

- Fast pattern recognition

$\Rightarrow$ Align FPGA code with trigger reconstruction

- Improved efficiency for off-axis tracks

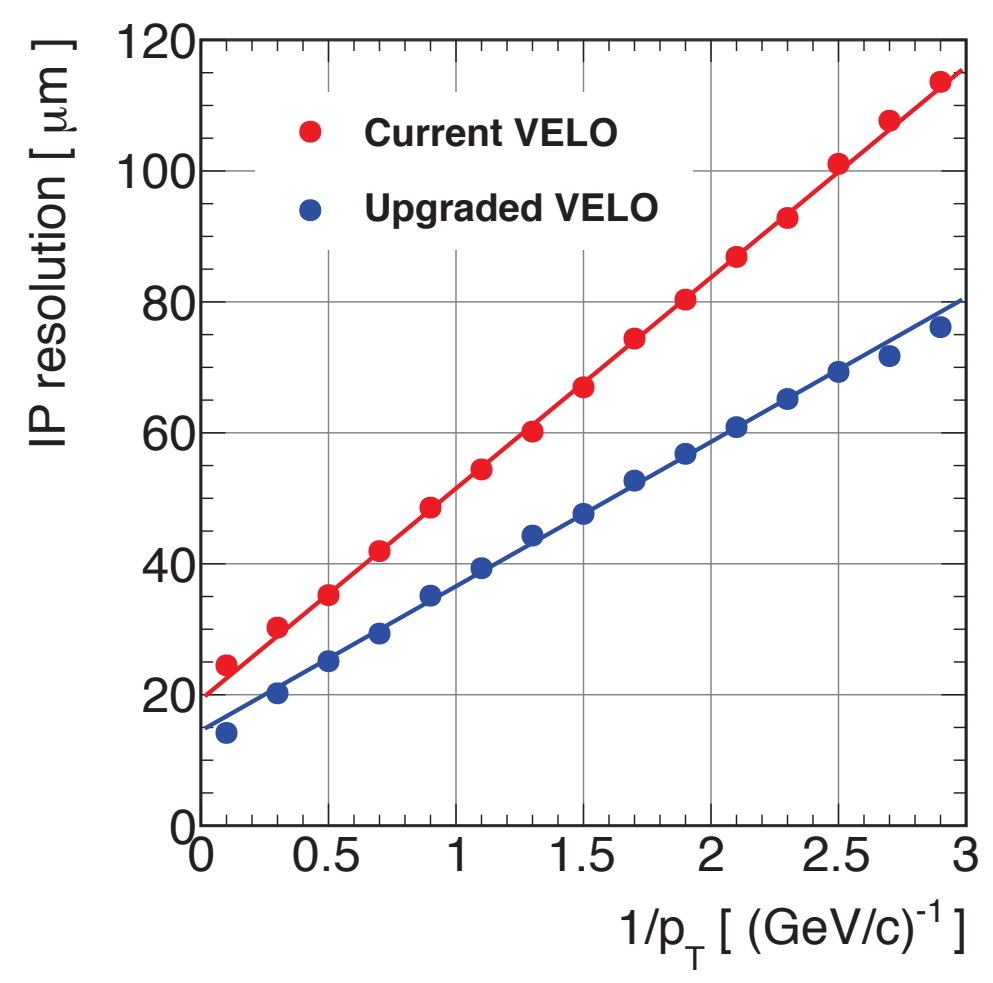


Table 16: Statistical sensitivities of the LHCb upgrade to key observables. For each observable the current sensitivity is compared to that which will be achieved by LHCb before the upgrade, and that which will be achieved with $50 \mathrm{fb}^{-1}$ by the upgraded experiment. Systematic uncertainties are expected to be non-negligible for the most precisely measured quantities. Note that the current sensitivities do not include new results presented at ICHEP 2012 or CKM2012.

\begin{tabular}{|c|c|c|c|c|c|}
\hline Type & Observable & $\begin{array}{l}\text { Current } \\
\text { precision }\end{array}$ & $\begin{array}{l}\mathrm{LHCb} \\
2018\end{array}$ & $\begin{array}{l}\text { Upgrade } \\
\left(50 \mathrm{fb}^{-1}\right)\end{array}$ & $\begin{array}{c}\text { Theory } \\
\text { uncertainty }\end{array}$ \\
\hline \multirow[t]{3}{*}{$B_{s}^{0}$ mixing } & $2 \beta_{s}\left(B_{s}^{0} \rightarrow J / \psi \phi\right)$ & $0.10[138]$ & 0.025 & 0.008 & $\sim 0.003$ \\
\hline & $2 \beta_{s}\left(B_{s}^{0} \rightarrow J / \psi f_{0}(980)\right)$ & $0.17[214]$ & 0.045 & 0.014 & $\sim 0.01$ \\
\hline & $a_{\mathrm{sl}}^{s}$ & $6.4 \times 10^{-3}[43$ & $0.6 \times 10^{-3}$ & $0.2 \times 10^{-3}$ & $0.03 \times 10^{-3}$ \\
\hline Gluonic & $2 \beta_{s}^{\text {eff }}\left(B_{s}^{0} \rightarrow \phi \phi\right)$ & $\overline{-}$ & 0.17 & 0.03 & 0.02 \\
\hline \multirow[t]{2}{*}{ penguins } & $2 \beta_{s}^{\mathrm{eff}}\left(B_{s}^{0} \rightarrow K^{* 0} \bar{K}^{* 0}\right)$ & - & 0.13 & 0.02 & $<0.02$ \\
\hline & $2 \beta^{\mathrm{eff}}\left(B^{0} \rightarrow \phi K_{S}^{0}\right)$ & $0.17[43]$ & 0.30 & 0.05 & 0.02 \\
\hline \multirow{2}{*}{$\begin{array}{l}\text { Right-handed } \\
\text { currents }\end{array}$} & $2 \beta_{s}^{\mathrm{eff}}\left(B_{s}^{0} \rightarrow \phi \gamma\right)$ & - & 0.09 & 0.02 & $<0.01$ \\
\hline & $\tau^{\mathrm{eff}}\left(B_{s}^{0} \rightarrow \phi \gamma\right) / \tau_{B_{a}^{0}}$ & - & $5 \%$ & $1 \%$ & $0.2 \%$ \\
\hline \multirow{4}{*}{$\begin{array}{c}\text { Electroweak } \\
\text { penguins }\end{array}$} & $S_{3}\left(B^{0} \rightarrow K^{* 0} \mu^{+} \mu^{-} ; 1<q^{2}<6 \mathrm{GeV}^{2} / c^{4}\right)$ & $0.08[67]$ & 0.025 & 0.008 & 0.02 \\
\hline & $s_{0} A_{\mathrm{FB}}\left(B^{0} \rightarrow K^{* 0} \mu^{+} \mu^{-}\right)$ & $25 \% 67$ & $6 \%$ & $2 \%$ & $7 \%$ \\
\hline & $A_{\mathrm{I}}\left(K \mu^{+} \mu^{-} ; 1<q^{2}<6 \mathrm{GeV}^{2} / c^{4}\right)$ & $0.25[76$ & 0.08 & 0.025 & $\sim 0.02$ \\
\hline & $\mathcal{B}\left(B^{+} \rightarrow \pi^{+} \mu^{+} \mu^{-}\right) / \mathcal{B}\left(B^{+} \rightarrow K^{+} \mu^{+} \mu^{-}\right)$ & $25 \% 85$ & $8 \%$ & $2.5 \%$ & $\sim 10 \%$ \\
\hline Higgs & $\mathcal{B}\left(B_{s}^{0} \rightarrow \mu^{+} \mu^{-}\right)$ & $1.5 \times 10^{-9}[13]$ & $0.5 \times 10^{-9}$ & $0.15 \times 10^{-9}$ & $0.3 \times 10^{-9}$ \\
\hline penguins & $\mathcal{B}\left(B^{0} \rightarrow \mu^{+} \mu^{-}\right) / \mathcal{B}\left(B_{s}^{0} \rightarrow \mu^{+} \mu^{-}\right)$ & - & $\sim 100 \%$ & $\sim 35 \%$ & $\sim 5 \%$ \\
\hline Unitarity & $\gamma\left(B \rightarrow D^{(*)} K^{(*)}\right)$ & $\sim 10-12^{\circ}[244,258]$ & $4^{\circ}$ & $0.9^{\circ}$ & negligible \\
\hline triangle & $\gamma\left(B_{s}^{0} \rightarrow D_{s} K\right)$ & - & $11^{\circ}$ & $2.0^{\circ}$ & negligible \\
\hline angles & $\beta\left(B^{0} \rightarrow J / \psi K_{\mathrm{s}}^{0}\right)$ & $0.8^{\circ}[43]$ & $0.6^{\circ}$ & $0.2^{\circ}$ & negligible \\
\hline Charm & $A_{\Gamma}$ & $2.3 \times 10^{-3}[43]$ & $0.40 \times 10^{-3}$ & $.07 \times 10^{-}$ & - \\
\hline$C P$ violation & $\Delta \mathcal{A}_{C P}$ & $2.1 \times 10^{-3}[18$ & $0.65 \times 10^{-3}$ & $12 \times 1$ & - \\
\hline
\end{tabular}




\section{Charm CP violation}

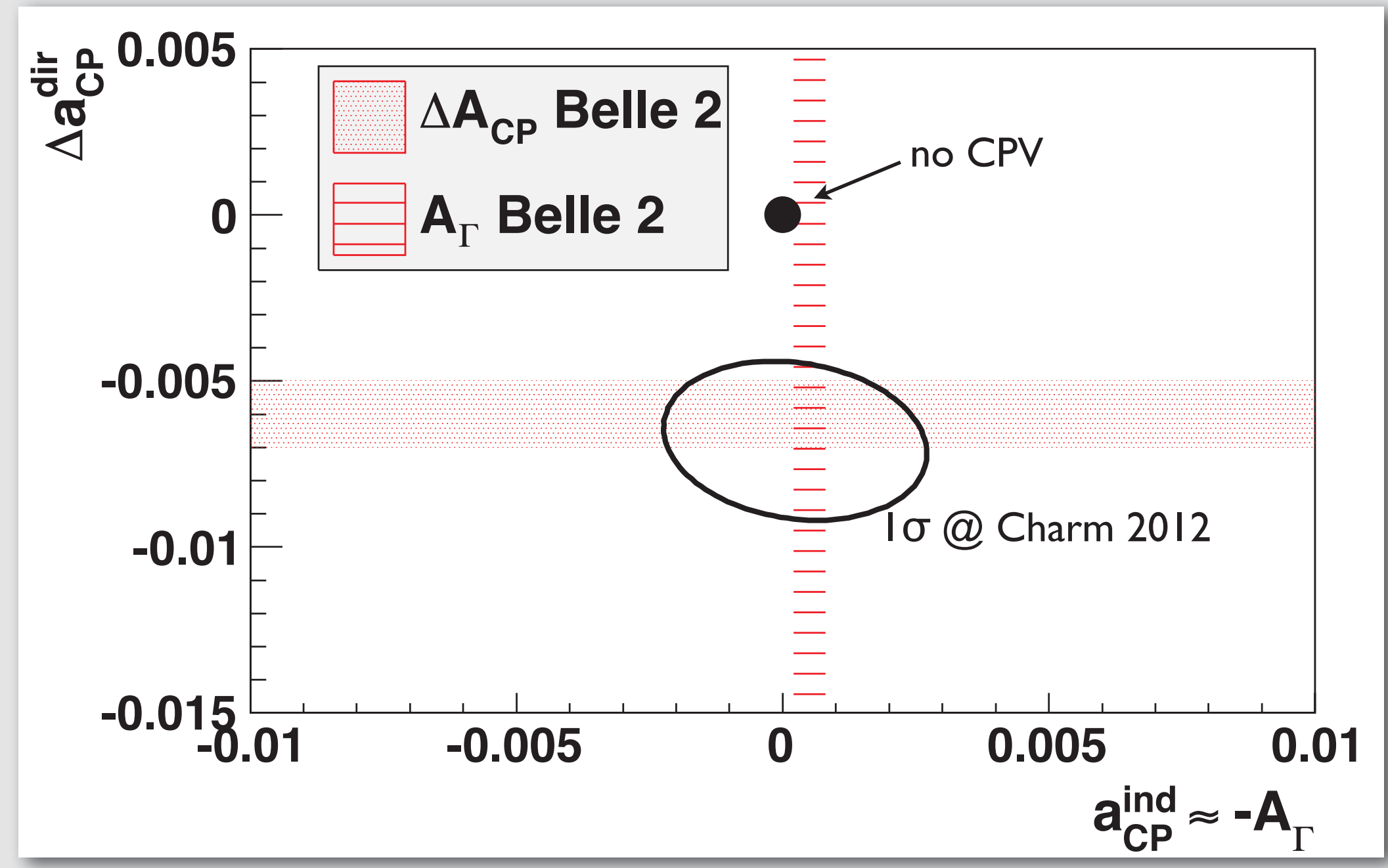




\section{Charm CP violation}



sub- $10^{-4}$ precision! 


\section{Charm CP violation}

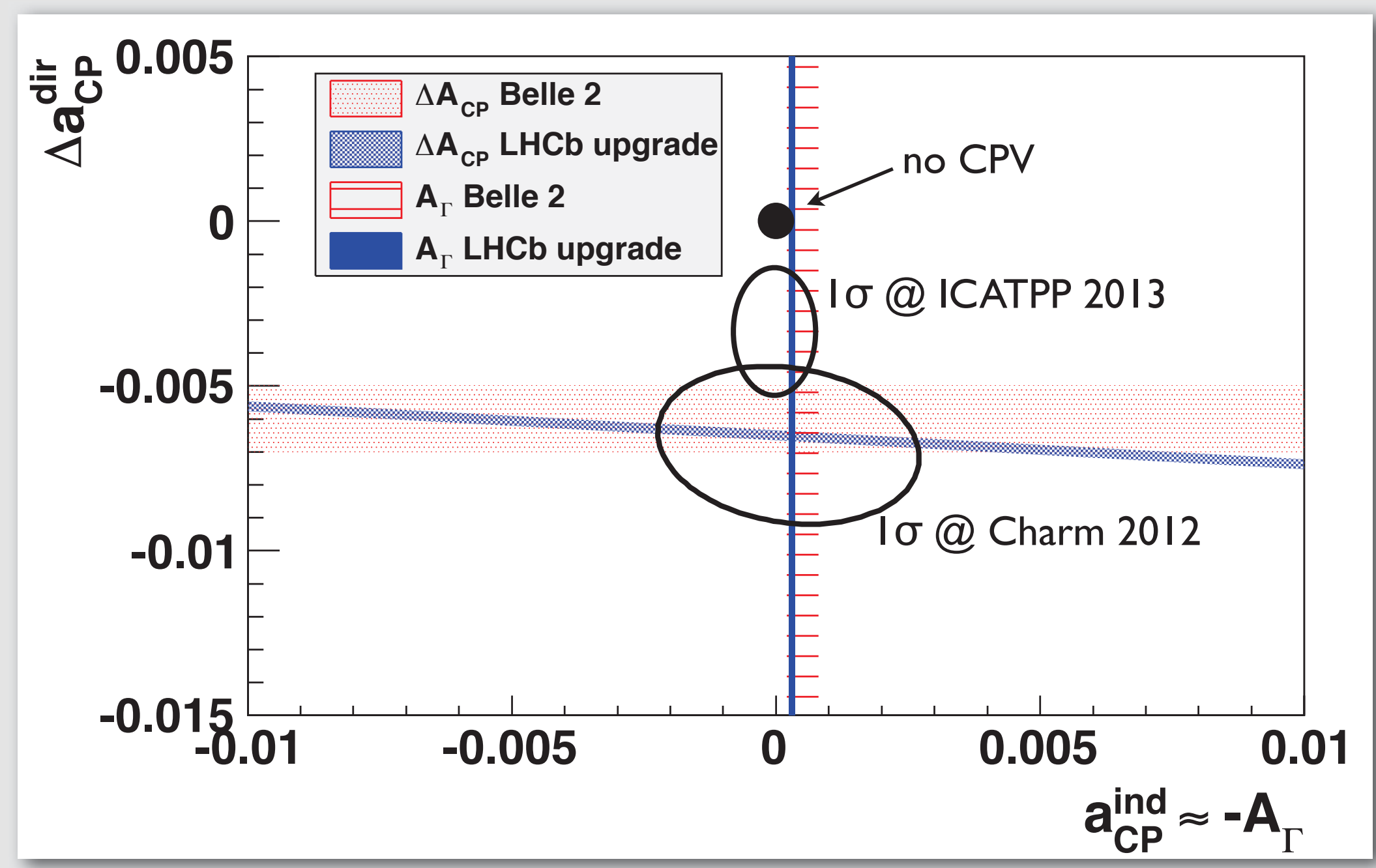

sub- $10^{-4}$ precision! 


\section{Conclusions}

- VELO detector

$\Rightarrow$ Reliable high performance vertexing for LHCb

$\Rightarrow$ Radiation damage following expectations

$\Rightarrow$ Second metal layer effects still tolerable

- VELO upgrade

$\Rightarrow$ Pixel detector with VeloPix readout at $40 \mathrm{MHz}$

- Microchannel cooling: first tests very promising

- New RF shield production mechanisms under study

$\Rightarrow$ VELO upgrade TDR end 2013 
MANCHESTER

1824

The University of Manchester

Backup 

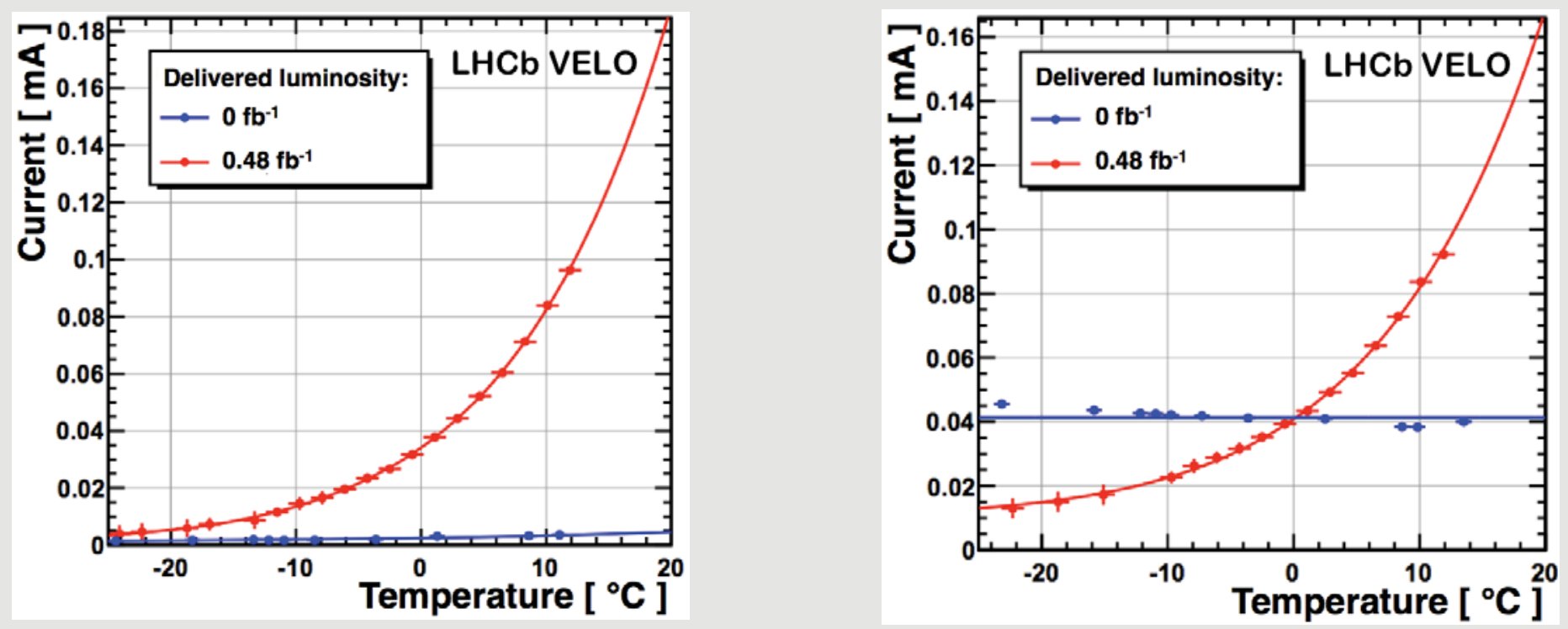


\section{MANCHESTER}

1824

The University of Manchester

\section{Current VELO IP}
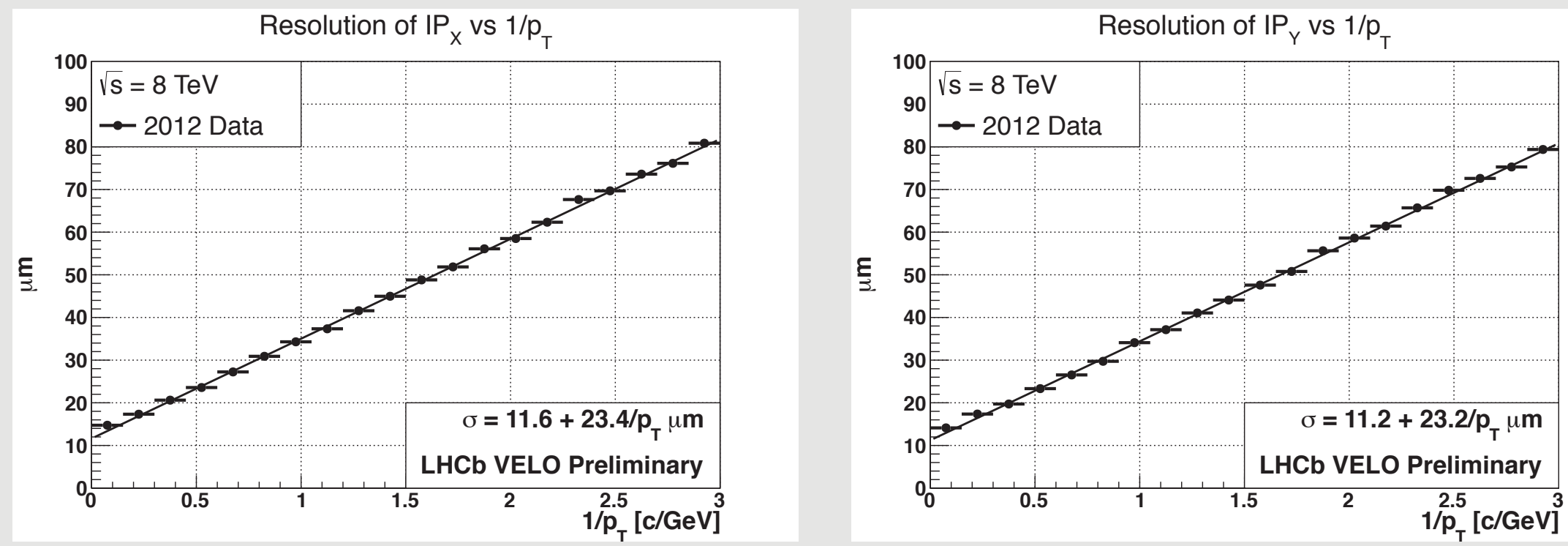
MANCHESTER

1824

\section{Bsmumu}

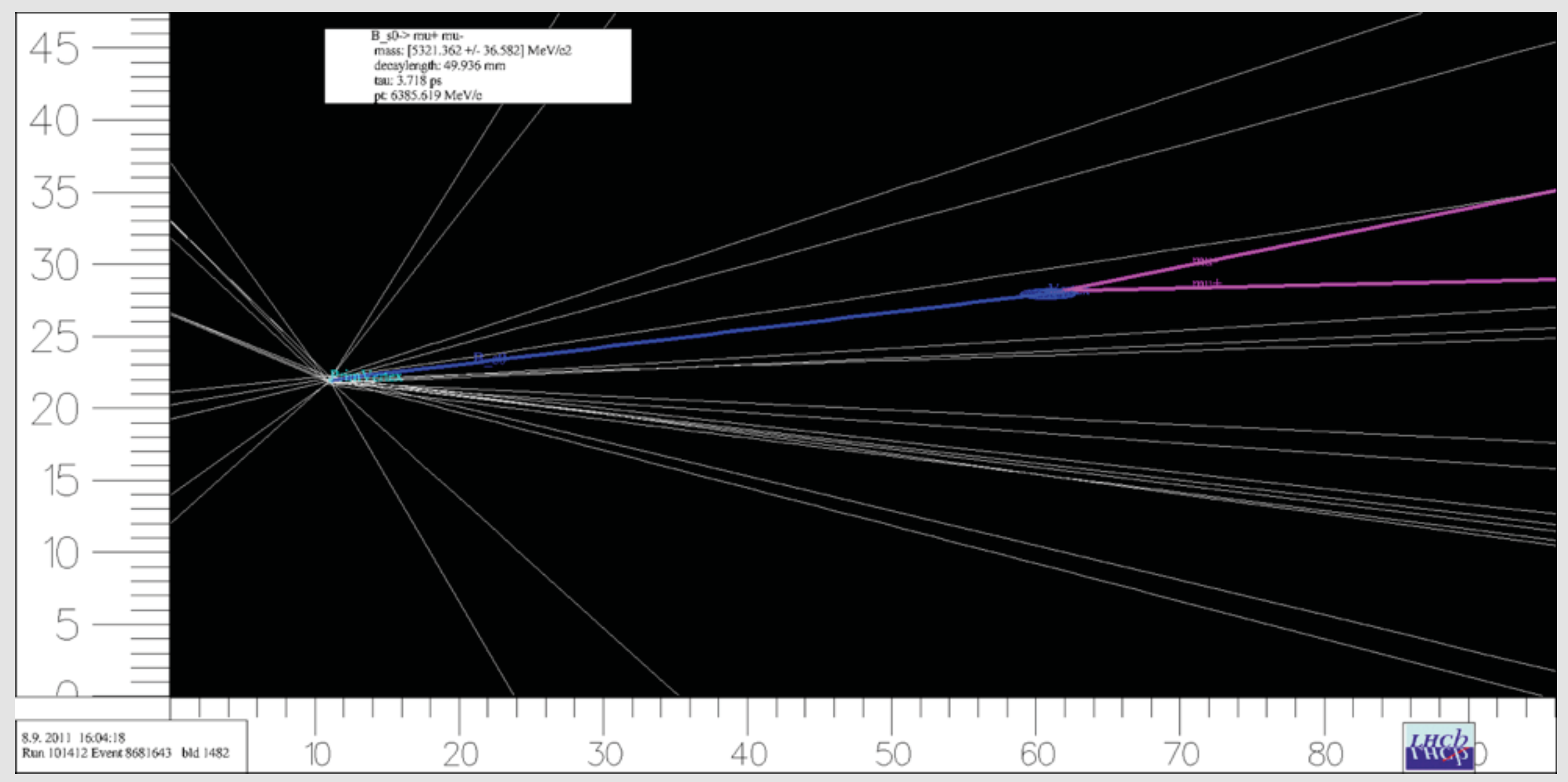


Tracking New silicon trackers Reduce straw coverage Fibre tracker

\section{$\mathrm{RICH}$}

New photon detectors

\section{Calorimeter+Muon} Remove MI, SPD, PS

New calorimeter FE electronics

Vertex Locator

a) New pixel detector b) Improved strip detector 
- Letter of Intent submitted March 20 I I $\rightarrow$ Physics case fully endorsed by LHCC

- FrameworkTDR submitted in June 2012

$\rightarrow$ Defining cost, milestones and institutes' interests

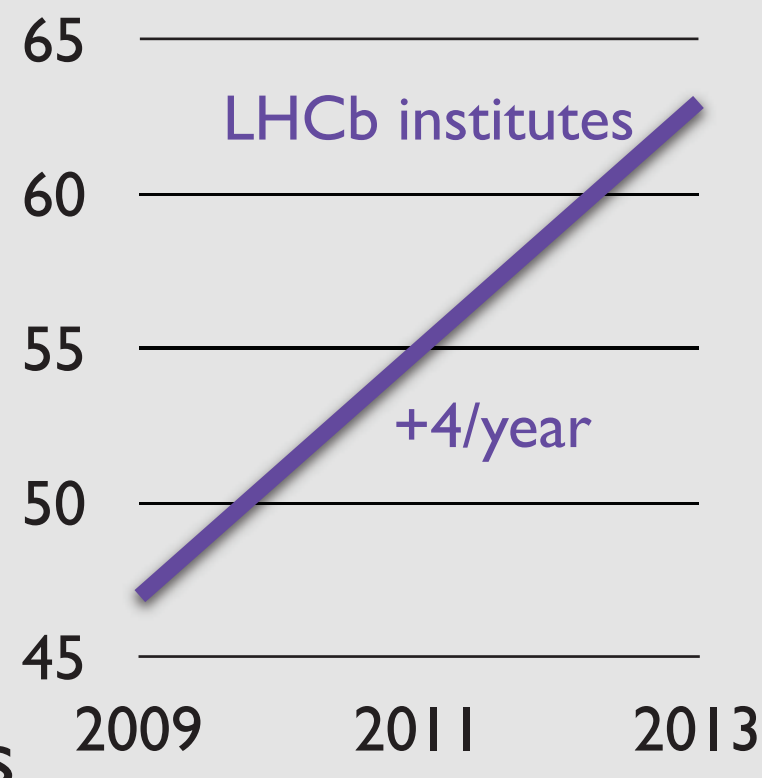

- Resource Review Board, April 2013

$\rightarrow$ First positive signals from several big funding agencies

- TDRs: end 2013

$\rightarrow$ Technical choices

$\mathrm{LHCl}$ CERv/HCC 2012-007

- Production and QA

$\rightarrow$ 20I4-20I7

- Installation in 2018

$\rightarrow$ Use LHC LS2

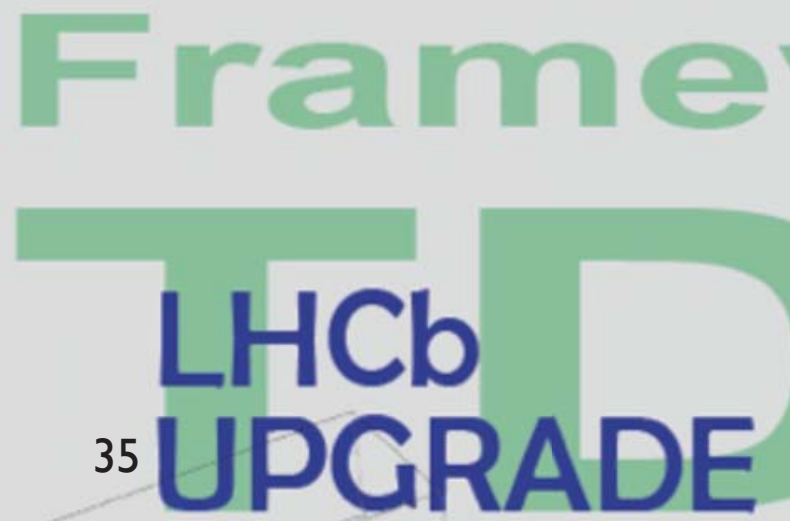

